\title{
RGS2-mediated translational control mediates cancer cell dormancy and tumor relapse
}

\author{
Jaebeom Cho, ${ }^{1}$ Hye-Young Min, ${ }^{1,2}$ Ho Jin Lee, ${ }^{1}$ Seung Yeob Hyun, ${ }^{1}$ Jeong Yeon Sim, $, 1,3$ Myungkyung Noh, ${ }^{1}$ Su Jung Hwang, ${ }^{4}$ \\ Shin-Hyung Park, ${ }^{2}$ Hye-Jin Boo, ${ }^{1,2}$ Hyo-Jong Lee, ${ }^{4}$ Sungyoul Hong, ${ }^{2}$ Rang-Woon Park, ${ }^{5}$ Young Kee Shin, ${ }^{2,3}$ Mien-Chie Hung, ${ }^{6,7}$ \\ and Ho-Young Lee ${ }^{1,2}$ \\ 'Creative Research Initiative Center for Concurrent Control of Emphysema and Lung Cancer, College of Pharmacy, ${ }^{2}$ College of Pharmacy and Research Institute of Pharmaceutical Sciences, and ${ }^{3}$ Department \\ of Molecular Medicine and Biopharmaceutical Sciences, Graduate School of Convergence Science and Technology and College of Pharmacy, Seoul National University, Seoul, Republic of Korea. ${ }^{4}$ College of \\ Pharmacy, Inje University, Gimhae, Gyungnam, Republic of Korea. ${ }^{5}$ Department of Biochemistry and Cell Biology, School of Medicine, and Cell \& Matrix Research Institute, Kyungpook National University, \\ Daegu, Republic of Korea. 'Graduate Institute of Biomedical Sciences, Research Center for Cancer Biology, and Center for Molecular Medicine, China Medical University, Taichung, Taiwan. 'Department of \\ Biotechnology, Asia University, Taichung, Taiwan.
}

\begin{abstract}
Slow-cycling/dormant cancer cells (SCCs) have pivotal roles in driving cancer relapse and drug resistance. A mechanistic explanation for cancer cell dormancy and therapeutic strategies targeting SCCs are necessary to improve patient prognosis, but are limited because of technical challenges to obtaining SCCs. Here, by applying proliferation-sensitive dyes and chemotherapeutics to non-small cell lung cancer (NSCLC) cell lines and patient-derived xenografts, we identified a distinct SCC subpopulation that resembled SCCs in patient tumors. These SCCs displayed major dormancy-like phenotypes and high survival capacity under hostile microenvironments through transcriptional upregulation of regulator of $G$ protein signaling 2 (RGS2). Database analysis revealed RCS2 as a biomarker of retarded proliferation and poor prognosis in NSCLC. We showed that RCS2 caused prolonged translational arrest in SCCs through persistent eukaryotic initiation factor 2 (elF2 $\alpha$ ) phosphorylation via proteasome-mediated degradation of activating transcription factor 4 (ATF4). Translational activation through RGS2 antagonism or the use of phosphodiesterase 5 inhibitors, including sildenafil (Viagra), promoted ER stressinduced apoptosis in SCCs in vitro and in vivo under stressed conditions, such as those induced by chemotherapy. Our results suggest that a low-dose chemotherapy and translation-instigating pharmacological intervention in combination is an effective strategy to prevent tumor progression in NSCLC patients after rigorous chemotherapy.
\end{abstract}

\section{Introduction}

Non-small cell lung cancer (NSCLC) is the leading cause of cancer-related death worldwide (1). Conventional chemotherapy is currently the standard treatment option for NSCLC (2). Although some primary tumors regress in response to therapy (3), a minority of cancer cells with chemoresistance reinitiate malignant tumors, resulting in poor outcomes $(4,5)$. Conventional chemotherapy targets actively cycling cancer cells (ACCs); however, it is a small subpopulation of slow-cycling/dormant cancer cells (SCCs) that seems to be the culprit of chemoresistance and cancer progression $(6,7)$. In cancer cells, dormancy - a nonproliferative or slowly proliferating state with reversible cell cycle arrest in $G_{0}$ phase (8) - is achieved via a quiescence program that induces p38/MAPK activation and ERK/ MAPK and PI3K/Akt inactivation $(9,10)$ and decreases mRNA translation (11), glucose uptake, and glycolysis (12).

One of the proposed features of SCCs is the adaptation to various hazardous stimuli, such as hypoxia and low glucose that evoke ER stress (13), and ER stress signaling has important roles in cancer

Conflict of interest: The authors have declared that no conflict of interest exists. Copyright: (c) 2021, American Society for Clinical Investigation.

Submitted: January 27, 2020; Accepted: September 9, 2020; Published: January 4, 2021

Reference information: J Clin Invest. 2021;131(1):e136779.

https://doi.org/10.1172/JCl136779. development and chemoresistance (14). ER stress stimulates the unfolded protein response (UPR), which ultimately controls the survival, growth arrest, or death decision of stressed cells depending on the duration and/or severity of the stress (15). UPR is mediated by 3 specific ER membrane proteins: activating transcription factor 6 alpha (ATF6 $\alpha$ ), inositol requiring enzyme 1 alpha (IRE1 $\alpha$ ), and protein kinase R-like ER kinase (PERK) (16). IRE1 $\alpha$-mediated and ATF6-mediated transcriptional programs induce the expression of genes involved in ER protein folding or degradation, resulting in protein clearance from the ER $(16,17)$. PERK-mediated phosphorylation of eukaryotic initiation factor 2 (eIF2 $\alpha$ ) transiently attenuates protein synthesis (17), leading to a reduction in the unfolded protein load. Subsequent ATF4 translation induces transcriptional activation of PPP1R15A/GADD34 (encoding GADD34, an adaptor for eIF2 $\alpha$ phosphatase PP1c that regulates eIF2 $\alpha$ dephosphorylation) or DDIT3/ CHOP/GADD153 (encoding GADD153/CHOP, a mediator of ER stress-induced apoptosis), leading to antiapoptotic effects through reversion of transient translational inhibition or apoptotic cell death (16). However, overload in protein synthesis induced by ATF4 overexpression was found to cause ER stress-induced apoptosis through ROS production (18). SCCs are proposed to acquire additional mechanisms for their survival against ER stress. For instance, upregulation of BiP and activation of PERK by p38-mediated pathway were proposed to protect SCCs from chemotherapy-induced ER stress 
(19). However, the phenotypical and functional features of SCCs and the molecular mechanisms underlying these features are largely unknown, especially in NSCLC, mainly because of the technical hurdles involved in isolating this biologically discrete subpopulation.

In the current study, we aimed to establish an experimental model of SCCs, understand the biology of cancer cell dormancy, and develop potentially novel SCC-targeting therapeutic strategies for patients with NSCLC. We isolated NSCLC SCCs from NSCLC cell lines and patient tumors by using proliferation-sensitive dyes and chemotherapeutic drugs. Our surrogate SCCs retained important characteristics of dormant cancer cells and transcriptional upregulation of regulator of $\mathrm{G}$ protein signaling 2 (RGS2). Analyses of publicly available data sets and tissue microarray (TMA) revealed significant correlations between the expression levels of RGS2 and those of cell cycle regulators in NSCLC. RGS2 overexpression was also associated with the poor prognosis of patients with NSCLC. Previous studies suggest that RGS2 acts as a stress response element (20-22) through GTPase-activating protein-dependent (GAP-dependent) and GAP-independent mechanisms (23, 24). Here, we demonstrated a potentially novel function of RGS2 that provides SCCs dormancy-like phenotypes and survival potential against ER stress through disruption of ATF4-mediated translational control. We showed that stimulation of protein synthesis in combination with chemotherapy is a potentially novel strategy to eliminate SCCs, thereby suppressing tumor progression in NSCLC.

\section{Results}

RGS2 is upregulated in SCCs within NSCLC cell lines, patient-derived xenograft tumors, and patient tumor tissues. Despite their dormant properties, a subpopulation of SCCs, characterized by chemoresistance and lack of proliferation marker Ki67 expression (6), has been found even in rapidly growing tumors (25) and cancer cell lines $(26,27)$. Therefore, we attempted to obtain SCCs by adapting the use of proliferation-dependent fluorescent cell-tracking dye CFSE that distinguishes subpopulations of slowly and rapidly growing cells (28). Three NSCLC cell lines (H460, H1299, and SK-MES-1) and a patient-derived xenograft (PDX) tumor (PDX1-1, PDX1-2; 2 different parts to account for tumor heterogeneity) were labeled with CFSE (Figure 1A). Over the course of 1 week, dividing cells progressively dilute out label and decrease in fluorescence intensity. On day 7, a rare subpopulation of label-retaining cells was distinguishable from unlabeled/poorly labeled bulk cells, and the labeled cells were sorted by flow cytometry. The CFSE-labeled cells that fell in the upper (>90\%), middle, or lower $(<10 \%)$ range were chosen as $\mathrm{CFSE}^{\mathrm{hi}}$, CFSE ${ }^{\mathrm{mid}}$, and $\mathrm{CFSE}^{\text {lo }}$ populations as previously described (29) (Figure 1B and Supplemental Figure 1A; supplemental material available online with this article; https:// doi.org/10.1172/JCI136779DS1). Compared with the CFSE ${ }^{\text {lo }}$ and $\mathrm{CFSE}^{\text {mid }}$ populations, $\mathrm{CFSE}^{\text {hi }}$ cells exhibited a significantly lower proliferation rate, Ki67 proliferation index (PI), a marker of $\mathrm{G}_{0}$ exit (Figure 1C and Supplemental Figure 1, B and C), mitotic index (Figure 1D), colony-forming capacity (Figure 1E), and chemosensitivity (Figure $1 \mathrm{~F}$ ). The $\mathrm{CSFE}^{\mathrm{mid}}$ population, wherein the majority of cells fell, showed a similar or slightly lower proliferation, mitotic index, anchorage-dependent colony-forming ability, or paclitaxel $(\mathrm{Pc})$ sensitivity compared with the $\mathrm{CSFE}^{\mathrm{lo}}$ population. Additional CFSE ${ }^{\text {hi }}$ SCCs from 2 different PDX tumors also showed significantly lower Ki67 PI, mitotic index, colony-forming capacity, and chemosensitivity compared with the corresponding CFSE ${ }^{\text {lo }}$ or $\mathrm{CFSE}^{\mathrm{mid}}$ populations (Supplemental Figures 1, C and D). All the $\mathrm{CFSE}^{\text {hi }}$ and $\mathrm{CFSE}^{\mathrm{lo}}$ populations eventually developed tumors in mice by the day they were euthanized, but the $\mathrm{CFSE}^{\text {hi }}$ population showed delayed tumor onset compared with the CFSE ${ }^{\mathrm{lo}}$ populations (Figure 1G). These results indicated that the minor CSFE ${ }^{\text {hi }}$ population was a distinct slow-cycling subpopulation.

We assessed the putative SCC-associated biomarkers by analyzing gene expression profiles of the $\mathrm{CFSE}^{\text {hi }}$ population versus their corresponding $\mathrm{CFSE}^{\mathrm{lo}}$ population. RNA-Seq analysis revealed that 319 genes were consistently upregulated or downregulated in the $5 \mathrm{CFSE}^{\text {hi }}$ populations compared with their corresponding $\mathrm{CFSE}^{\text {lo }}$ populations (|fold change $\mid \leq 1.25$, Figure $2 \mathrm{~A}$ and Supplemental Figure 2A). Gene ontology (GO) analysis of these upregulated genes revealed that various genes, including those involved in the negative regulation of the response to stimuli or the regulation of cell proliferation, were significantly enriched in the CFSE ${ }^{\text {hi }}$ population (Figure 2B). In contrast, the commonly downregulated genes did not show such correlations. Further analysis of the commonly upregulated genes in the CFSE ${ }^{\text {hi }}$ populations and the gene products associated with the regulation of proliferation and the cell cycle revealed consistent upregulation of 11 genes (RGS2, PRDM11, CCPG1, CLU, IER5, SIX3, MAP3K8, PDCD2L, ZNF703, $B C L 6$, and LAMA5) (Supplemental Figure 2B). By performing real-time PCR analysis, we validated upregulation of these genes' expression in $\mathrm{CFSE}^{\text {hi }}$ populations compared with their corresponding $\mathrm{CFSE}^{\text {lo }}$ populations. We analyzed 4 publicly available data sets and found that, among 11 upregulated genes, only RGS2 expression was consistently and significantly correlated with the expression of proliferation-associated genes (Supplemental Table 1). Upregulation of RGS2 transcription was observed in all CFSE ${ }^{\text {hi }}$ SCCs from 3 NSCLC cell lines and 4 PDX tumors (fold change $=2.5$, on average of 7 CFSE $^{\text {hi }}$ SCCs) (Figure 2C). We confirmed our findings by using the PKH26 dye, which was incorporated into the plasma membrane (30) (Supplemental Figure 3A). The PI of CSFE-labeled cells was comparable with that of PKH26-labeled cells (31). Compared with the $\mathrm{PKH} 26^{\text {lo }}$ populations, the $\mathrm{PKH} 26^{\text {hi }}$ populations displayed downregulated proliferation, decreased Ki67 PI and colonyforming ability, resistance to Pc-induced cell death, and elevated RGS2 mRNA expression (Supplemental Figure 3, B-E).

RGS2 expression negatively correlates with the expression of genes encoding cell proliferation and is positively associated with poor clinical outcomes in patients with NSCLC. We analyzed publicly available data sets to determine the impact of RGS2 expression in clinical outcomes of patients with NSCLC. Although no significant correlation was observed between RGS2 mRNA expression and NSCLC histological subtype, patients with lung adenocarcinoma with elevated RGS2 mRNA expression exhibited significantly reduced overall and disease-free survival rates (Figure 2D and Supplemental Figure 4A). We then assessed the correlation between RGS2 expression and genes associated with regulation of cell proliferation and found a significant inverse correlation between the expression of RGS2 and that of genes involved in cell proliferation, including MKI67 (which encodes Ki67), PCNA, and CDK2 (Figure 2E and Supplemental Figure 4B). RGS2 expression also positively correlated with the expression of genes encoding cyclin-dependent kinase inhibitors, such as 
A

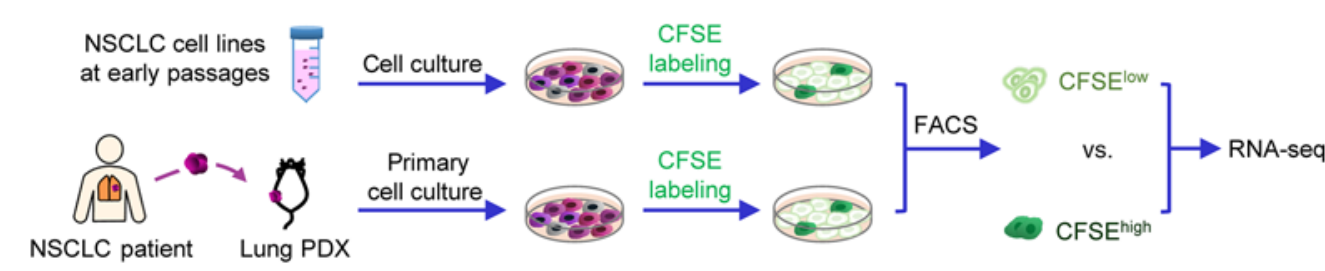

B

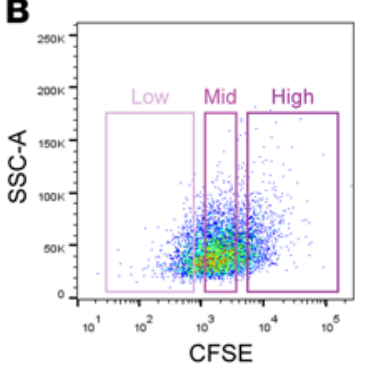

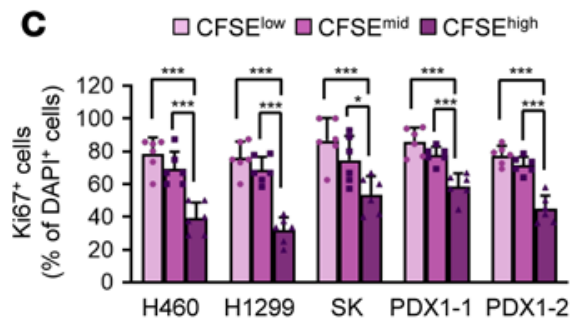

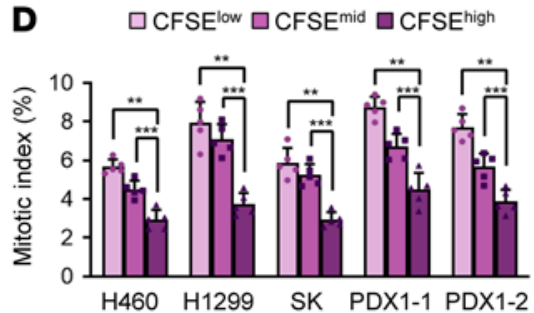

E $\quad \square C$ CFE $^{\text {low }} \square$ CFSE $^{\text {mid }} \square$ CFSE $^{\text {high }}$
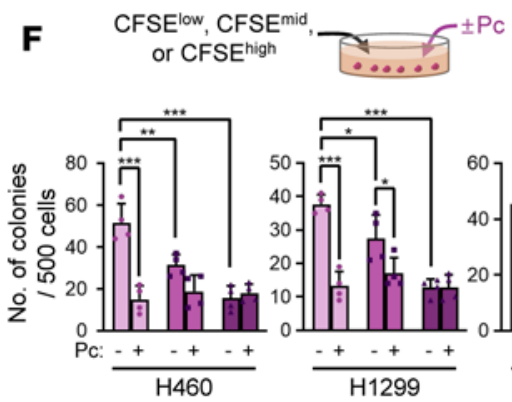

$\square C F S E^{\text {low }} \square C F S E^{\text {mid }} \square C F S E^{\text {high }}$

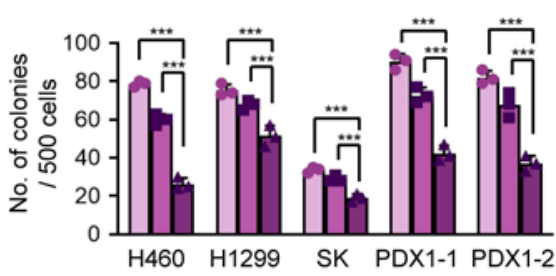

Figure 1. Isolation of slow-cycling and chemoresistant population in NSCLC cell line and lung PDX samples. (A) Schematic diagram distinguishing the intrinsic

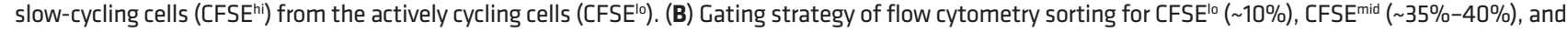
CFSE $^{\text {hi }}(\sim 10 \%)$ populations. (C-F) The basal Ki67 positivity (C), mitotic index (D), anchorage-dependent colony formation (E), and resistance to chemotherapy in the anchorage-independent colony formation (F) of CFSE ${ }^{\text {hi }}$ cells compared with CFSEmid and CFSE ${ }^{10}$ cells. (C) Delayed tumorigenesis of CFSE ${ }^{\text {hi }}$ cells compared with CFSE ${ }^{10}$ cells in NOD/SCID mice (H460 and PDX1-2, $n=5$ per group; H1299, SK [SK-MES-1], and PDX1-1, $n=6$ per group). The data are presented as the mean \pm SD. $n=6$ for $\mathbf{C} ; n=5$ for $\mathbf{D} ; n=3$ for $\mathbf{E} ; n=4$ for $\mathbf{F} .{ }^{*} P<0.05,{ }^{* *} P<0.01$, and ${ }^{* * *} P<0.001$, as determined by 1-way ANOVA with Dunnett's post hoc test (C, D, E, F).

CDKN1A and CDKN1B (Figure 2E and Supplemental Figure 4C). Gene set enrichment analysis (GSEA) (32) revealed that gene sets associated with proliferation were depleted in RGS $2^{\text {hi }}$ populations, whereas those associated with the response to a hazardous microenvironment, such as oxidative stress, hypoxia (33), and the UPR (34), were significantly enhanced in RGS2 $2^{\text {hi }}$ populations (FDR $<25 \%$ ) (Figure 2F and Supplemental Figure 5, A and B). We further validated the correlation between RGS2 expression and proliferation of NSCLC cells in patients by performing immunofluorescence (IF) analysis of an NSCLC TMA $(n=40)$. We observed a significant inverse correlation between RGS2 expression and Ki67 levels in the tissues (Figure $2 \mathrm{G}$ and Supplemental Table 2). Collectively, these results suggest that RGS2 is an important regulator of cellular dormancy and a clinically useful biomarker for poor prognosis in patients with NSCLC.

Repeated chemotherapy exposure enriches RGS2hi SCCs from NSCLC cell lines and PDX tumors. To investigate the role of RGS2 in functional features of NSCLC SCCs, we attempted to establish RGS2 $2^{\text {hi }}$ SCCs. Because of the lack of techniques that reliably identify functionally defined RGS2 ${ }^{\text {hi }}$ SCCs, we adapted the use of chemotherapeutic drugs that target ACCs. We cultured a panel of human NSCLC cell lines (H460, H1299, SK-MES-1, H226B, and H1792) in the presence of Pc, cisplatin (Cs), or pemetrexed (Pm), chemotherapeutic drugs that are frequently used in the clinic (3) (Figure 3A). After 3-4 months of selection by repeated exposure to and removal of the drugs (drug holidays), we found that although most of the cells died, small surviving subpopulations eventually formed colonies. These surviving subpopulations were highly chemoresistant, as shown by minimal changes in their viability, colony-forming capacity, and apoptotic activity in the presence of chemotherapeutic drugs (Supplemental Figure 6, A-C). We obtained 2 distinct groups of subpopulations. The first group (H226B/PcR, $\mathrm{H} 46 \mathrm{O} / \mathrm{PmR}$, and H1792/PmR) exhibited a proliferation rate and RGS2 expression similar to those of their respective parental cells (Supplemental Figure 7, A-C). By contrast, the second group (H460/ PcR, H1299/CsR, H1299/PmR, and SK-MES-1/PcR [SK/PcR]) showed significantly decreased proliferation and colony-forming capacities and transcriptional upregulation of RGS2 (Figure 3, B-D) compared with corresponding parental cells without detectable 
A

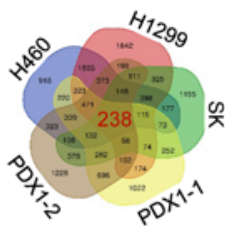

CFSEhigh vs. CFSElow

Upregulated genes (Fc $\geq 1.25$

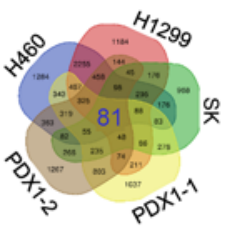

CFSEhigh vs. CFSElow

Downregulated genes $(\mathrm{Fc} \leq 0.8)$

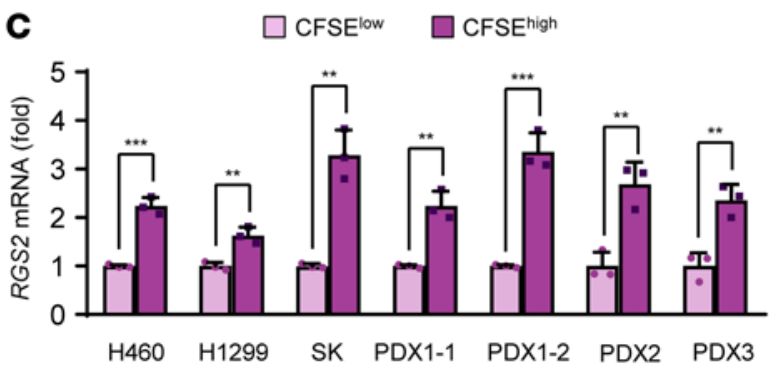

D
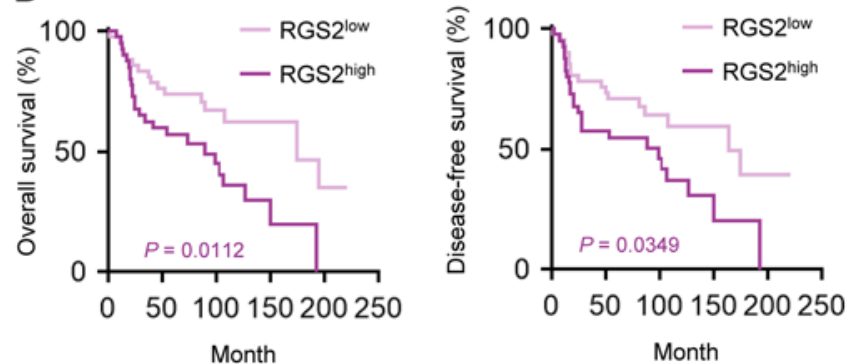

E
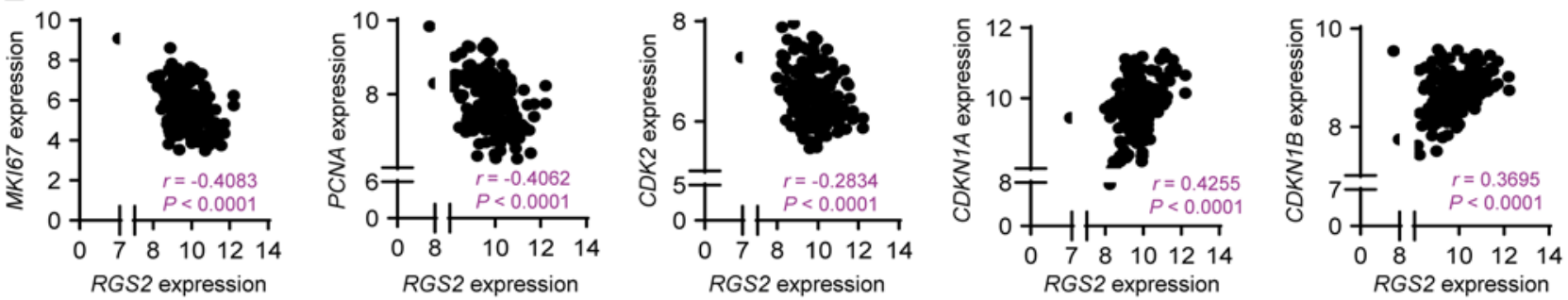

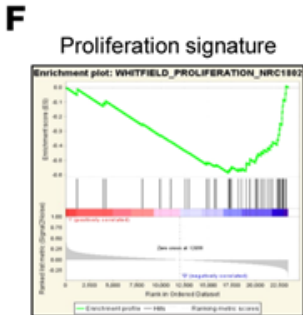

NES: $-1.31 /$ FDR: 0.25

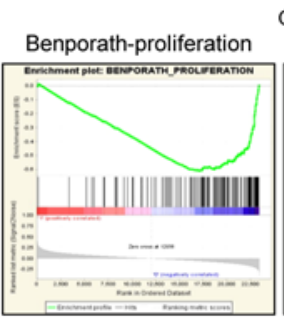

NES: $-1.57 / F D R: 0.094$

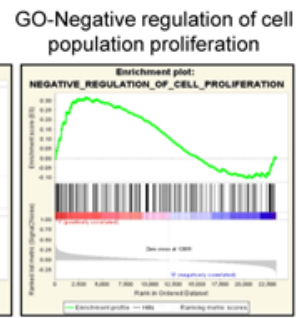

NES: 1.42 / FDR: 0.023

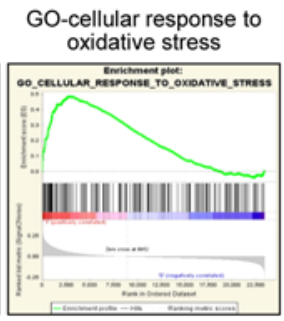

NES: 1.64 / FDR: 0.0

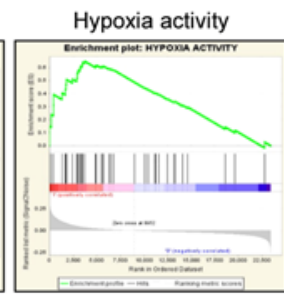

NES: 1.42 / FDR: 0.108

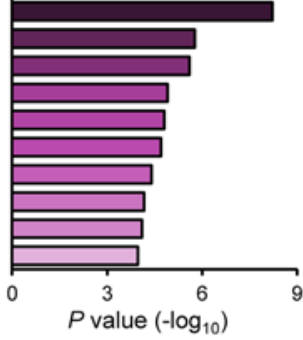

G
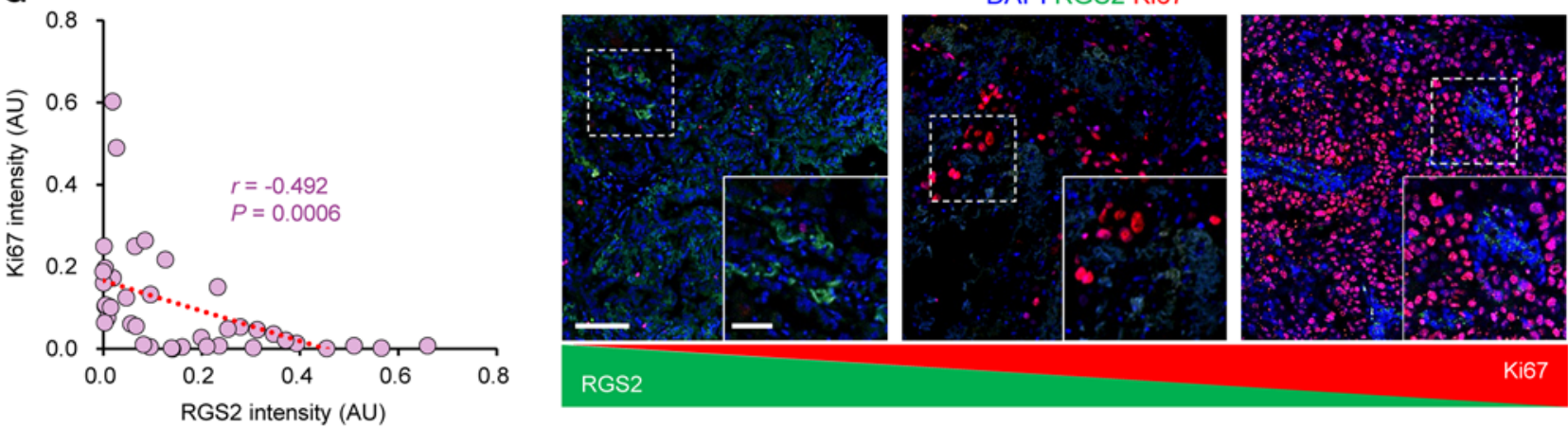

RGS2

Ki67

Figure 2. Identification of RGS2 as a potentially novel biomarker associated with quiescence-like phenotypes and the association of RGS2 expression with poor clinical outcomes. (A) Venn diagrams of differentially regulated genes in CFSE ${ }^{\text {hi }}$ cells compared with CFSE ${ }^{10}$ cells. (B) Gene Ontology (CO) terms significantly enriched in CFSE hi cells compared with CFSE ${ }^{\text {lo }}$ cells. (C) Upregulated RGS2 mRNA expression in the CFSE ${ }^{\text {hi }}$ populations. (D) Kaplan-Meier survival curve showing poor overall and disease-free survival in RGS2 $2^{\text {hi }}$ lung ADC $(n=42)$ compared with RGS2 $2^{10}$ lung ADC $(n=42)$ in a data set GSE30219. ADC: adenocarcinoma. (E) The correlation between RCS2 expression and cell proliferation-associated gene expressions in NSCLC tumors using a data set CSE63074. (F) GSEA to determine the enrichment of cell proliferation-, stress response-, or UPR-related gene sets in RGS2 ${ }^{\text {hi }}$ NSCLC using data set GSE3141. NES: normalized enrichment score. (C) Correlation analysis between RGS2 and Ki67 expression in a tissue microarray comprising 40 NSCLC tumors. Scale bar: $100 \mu \mathrm{m}$. Scale bar (inset): $50 \mu \mathrm{m}$. The data are presented as the mean \pm SD. $n=3$ for $\mathbf{C}$. ${ }^{*} P<0.01$, and ${ }^{* * *} P<0.001$, as determined by 2 -tailed Student's $t$ test (C) and log-rank test (D). Correlation was determined using Pearson's and Spearman's correlation (E and $\mathbf{G})$. 
signs of apoptotic cell death or autophagy (Supplemental Figure 8, A and B). Hence, we chose the second group for further analyses.

We assessed whether the subpopulations had label-retaining cells. To this end, the chemotherapy-resistant subpopulations and their corresponding parental cells were labeled with CSFE and then monitored for label-retaining overtime in culture. The chemotherapy-resistant subpopulations revealed obviously greater label-retaining than did their corresponding parental cells for up to 10 days (Supplemental Figure 8C). These subpopulations seemed to be in a state of quiescence rather than senescence, as evidenced by significant decreases in Ki67 PI, cyclin D1 and CDK4 expression and increases in p27 and p21 expression, p38 phosphorylation, and ERK1/2 dephosphorylation (Figure 3E and Supplemental Figure 8D) without detectable senescence-associated changes, including morphological alterations (i.e., cell flattening and vacuolization) or senescence-associated $\beta$-galactosidase (SA- $\beta$-gal) activation (35, 36) (Supplemental Figure $8 \mathrm{E}$ ). In addition, compared with their corresponding parental cells, these subpopulations also showed reductions in metabolic activity and mRNA translation, as evidenced by decreases in the oxygen consumption rate (OCR)/extracellular acidification rate (ECAR) and Renilla and firefly luciferase reporter activities, which reflect CAP-dependent and CAP-independent protein translation, respectively (37), and a decrease in methionine analog incorporation, which reflects de novo protein synthesis (Figure 3, F and G; and Supplemental Figure 8F). When tumorigenic capacity was analyzed in mice, H460/PcR, H1299/ CsR, and H1299/PmR cells successfully developed tumors with a markedly delayed tumor onset compared with their corresponding parental cells (Figure $3 \mathrm{H}$ ). Once developed, the tumors from these subpopulations displayed a steeply increased growth rate and level of chemoresistance (Figure $3 \mathrm{H}$ and Supplemental Figure 9). Hence, these subpopulations appeared to remain in a slow-cycling state until a permissive in vivo microenvironment supported recurrent tumor growth. Together, these results suggest that $\mathrm{H} 460 / \mathrm{PcR}$, H1299/CsR, H1299/PmR, and SK/PcR subpopulations represent a group of functional SCCs in NSCLC. Because the SK/PcR cells failed to generate xenograft tumors within 100 days after injection, we used the H460/PcR, H1299/CsR, and H1299/PmR subpopulations as preclinical models to study the biology of RGS2 ${ }^{\text {hi }}$ SCCs.

RGS2 promotes SCC quiescence-like phenotypes and survival against ER stress-induced apoptosis. To determine the role of RGS2 in quiescence-like phenotypes of SCCs, we used H460/PcR cells, in which RGS2 expression was silenced by stable transfection with shRNAs that targeted different regions of RGS2 mRNA (H460/ PcR-shRGS2), and H460 cells with forced overexpression of RGS2 (H460-RGS2). Compared with control cells (H460/PcR-shEV), 2 different H460/PcR-shRGS2 cells exhibited significant increases in proliferation, Ki67 PI, and colony-forming capacities (Figure 4A and Supplemental Figure 10, A-D). Moreover, H460/PcRshRGS2 displayed increased expression levels of cyclin D1, CDK4, and PCNA compared with H460/PcR-shEV cells (Figure 4B, left). Conversely, H460-RGS2 cells exhibited decreased levels of these proteins compared with control cells (H460-EV) (Figure 4B, right). Furthermore, compared with H460/PcR-shEV cells, H460/PcRshRGS2 cells displayed increases in the OCR and ECAR, CAP-dependent and CAP-independent protein translation, and de novo protein synthesis (Figure 4, C and D, left and Supplemental Figure
10 , $\mathrm{E}$ and $\mathrm{F}$ ), whereas all of these activities were decreased in $\mathrm{H} 460$ RGS2 cells compared with H460-EV cells (Figure 4, C and D, right, and Supplemental Figure 10F). These findings support that RGS2 expression endows SCCs with quiescence-like phenotypes.

Given that persistent ER stresses, such as DNA damage caused by chemotherapy (14), ER calcium depletion, and hypoxia (38), can induce apoptotic cell death $(16,39)$, we reasoned that SCCs might evolve unique mechanisms that ensure their survival during conditions that would otherwise cause ER stress-induced apoptosis. Indeed, SCCs displayed the capacity to escape ER stress-induced apoptosis, as shown by minimal changes in caspase-3 and PARP cleavage and the low number of sub-G1 phase cells in the presence of ER stressors thapsigargin (TG) and hypoxia (40) (Figure 4E and Supplemental Figure 10, G-I). Of note, compared with H460-EV cells, H460-RGS2 cells showed a significantly increased survival capacity against TG, hypoxia, and Pc (Figure 4F and Supplemental Figure 10J). Conversely, compared with H460/PcR-shEV cells, H460/PcR-shRGS2 cells showed a dramatically decreased survival capacity against TG- and Pc-induced stresses (Figure 4G and Supplemental Figure 10, K and L). Consistently, 2 different H460/ PcR cells, in which RGS expression was ablated by CRISPR/Cas9 system, also revealed significantly increased proliferation, Ki67 PI, anchorage-dependent and -independent colony-forming capacities, and protein translation, but underwent apoptosis in response to TG or Pc treatment (Supplemental Figure 11).

When inoculated in mice, H460/PcR-shRGS2 cells displayed markedly delayed tumor development compared with those inoculated with H460/PcR-shEV cells (Figure 4H). Specifically, every mouse inoculated with $\mathrm{H} 460 / \mathrm{PcR}-\mathrm{shEV}$ cells developed tumors by day 29, at which time only one of the mice inoculated with H460/ PcR-shRGS2 cells had developed tumors. Every mouse bearing H460/PcR-EV or H460/PcR-shRGS2 xenografts showed tumor formation by day 37; however, the H460/PcR-shRGS2 tumors were significantly smaller in volume than H460/PcR tumors (Figure 4I). Tumors inoculated with H460/PcR-shRGS2 xenografts also showed significantly retarded growth compared with those inoculated with $\mathrm{H} 460 / \mathrm{PcR}$ cells transfected with scrambled shRNA (Supplemental Figure 12). RGS2 thus seemed to endow SCCs with survival capacity, especially in an in vivo microenvironment featuring various ER stresses, such as hypoxia and glucose deprivation (41). These results suggest that RGS2 expression allows SCCs' survival capacity against ER stress-induced apoptosis.

ATF4 expression is suppressed in SCCs and remains unchanged during ER stress. We investigated the mechanisms that mediate the functional roles of RGS2 in SCCs. We reasoned that RGS2 expression may allow SCCs the capacities of cancer stem cells (CSCs), which by definition self-renew, display tumor-initiating potential, and induce drug resistance (42). However, CSC-associated functional features, including the expression of CSC- and epithelial-mesenchymal transition-related markers, sphere-forming ability, and aldehyde dehydrogenase positivity remained minimally changed in the established RGS2 ${ }^{\text {hi }}$ SCCs compared with their parental cells (Supplemental Figure 13). We next monitored the expression levels of genes involved in the PERK, ATF6, and IRE1 $\alpha$ branches of the UPR in SCCs. We found that ATF6, ERN1, and target gene signatures of the ATF6 and IRE1 $\alpha$ branches were almost consistently upregulated in the 3 SCCs compared with their corresponding parental cells (Figure 5A). These 

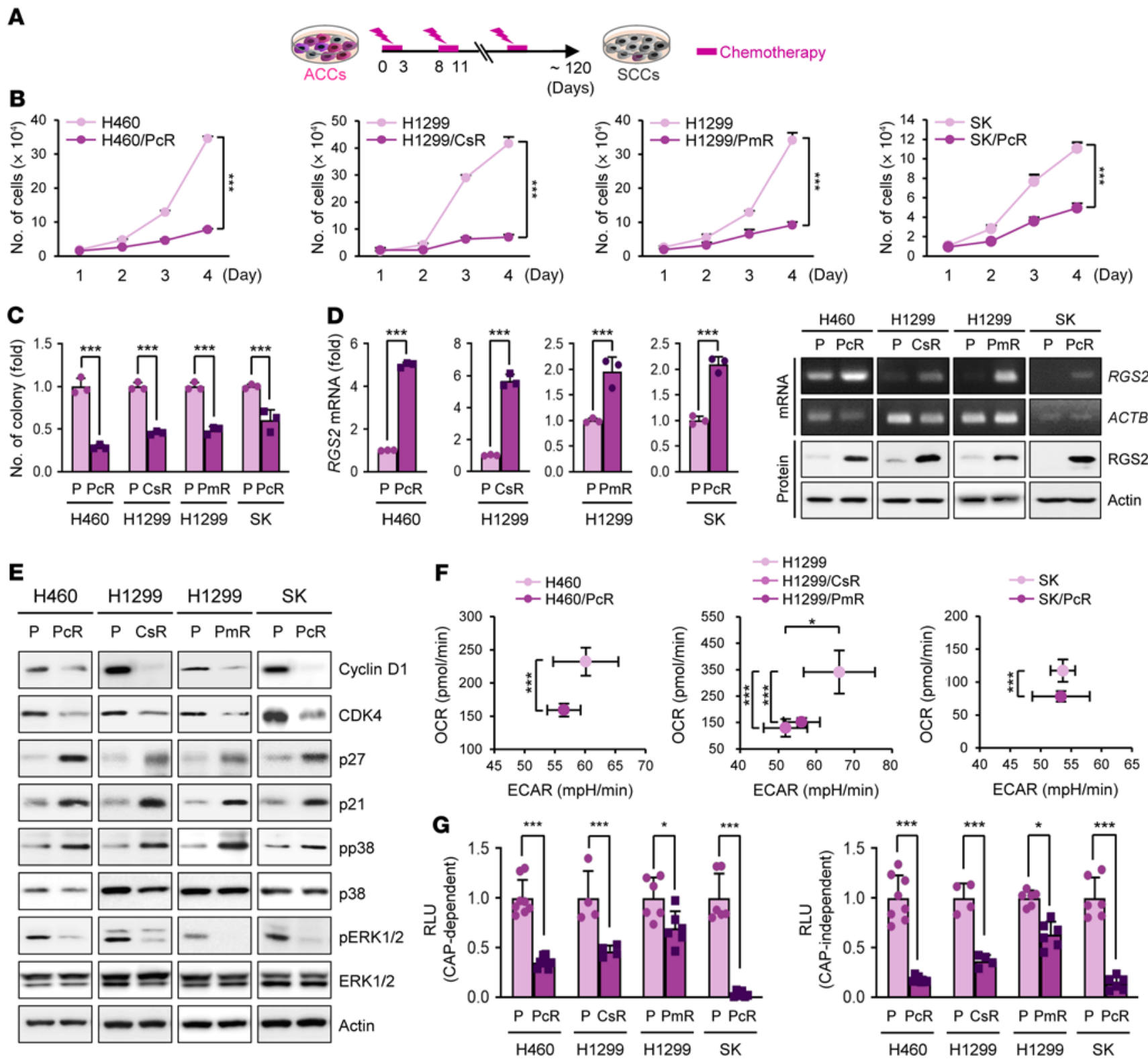

\section{$\mathbf{H}$}

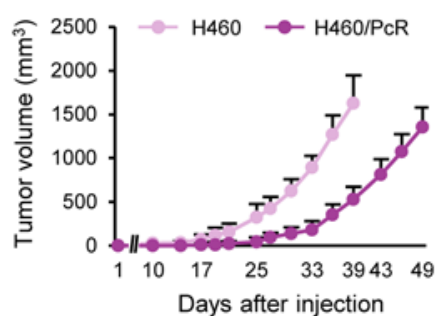

NSCLC cells or their SCCs

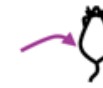

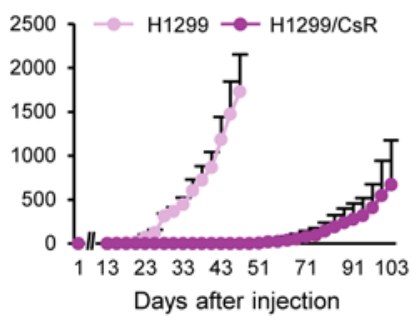
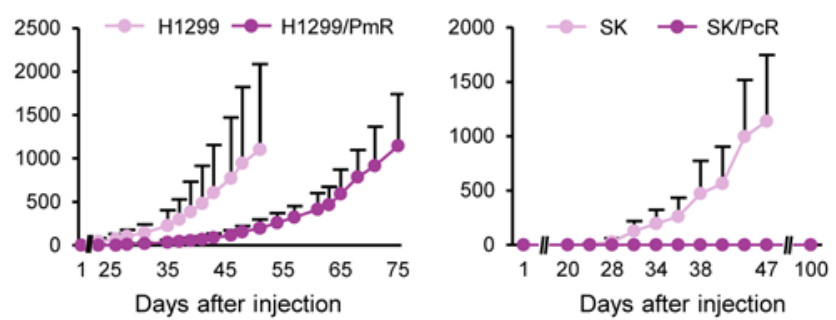

Figure 3. Quiescence-like phenotypes of SCC sublines. (A) Schematic diagram showing enrichment of SCCs (H460/PcR, H1299/CsR, H1299/PmR, and SK/ PcR) by long-term treatment with chemotherapeutic drugs to ACCs ( $4460, \mathrm{H} 1299$ and SK). (B and C) Downregulation of cell proliferation (B) and anchorage-dependent colony formation (C) of SCCs compared with the corresponding ACCs. (D) Upregulation of RCS2 mRNA and protein expression in SCCs compared with the ACCs. (E) Immunoblotting showing modulation of cell proliferation, quiescence, and cell cycle arrest-associated markers in SCCs compared with the ACCs. (F and $\mathbf{G})$ Downregulation of energy metabolism (F), CAP-dependent and -independent protein translation (C) in SCCs compared with ACCs. (H) Delayed growth of xenograft tumors generated from SCCs compared with those generated from ACCs in NOD/SCID mice (H460, $n=6, \mathrm{H} 460 / \mathrm{PCR}, n=6$; H1299, $n=6, \mathrm{H} 1299 / \mathrm{CsR} n=5$; H1299, $n=10, \mathrm{H1299} / \mathrm{PmR}, n=10 ; \mathrm{SK}, n=5$, SK/PcR, $n=5$ ). The data are presented as the mean \pm SD. $n=3$ for B, C, D, F; $n=$ 4 or 8 for $\mathbf{G} .{ }^{*} P<0.05,{ }^{*} P<0.01$, and ${ }^{* * *} P<0.001$, as determined by a 2 -tailed Student's $t$ test. 
A

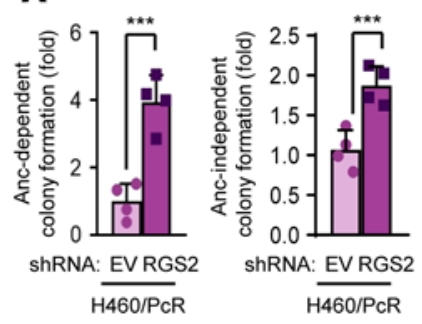

B

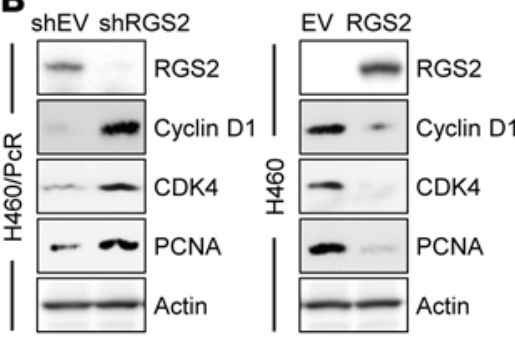

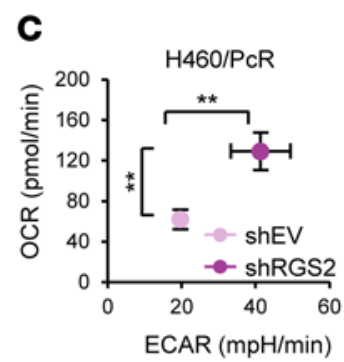

D
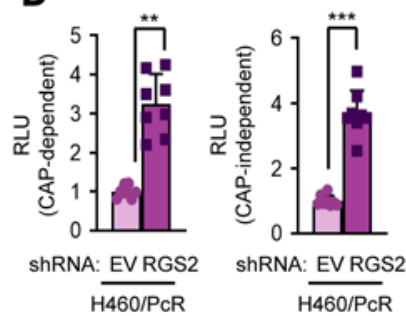

$\frac{\text { H460/PcR }}{\mathrm{H} 460 / \mathrm{PcR}}$
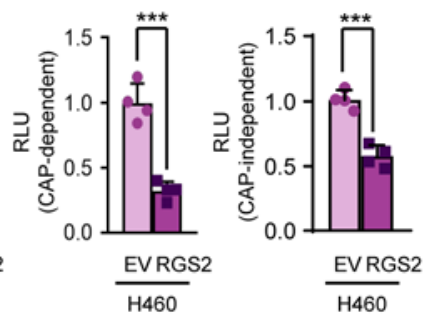

\section{E}
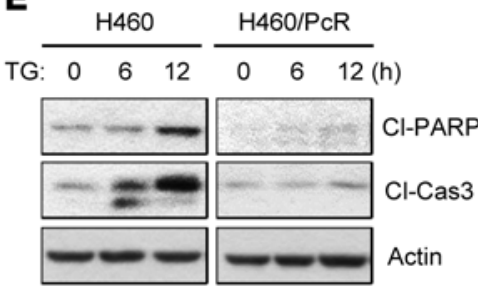

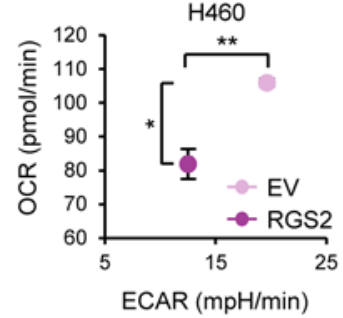

\section{$\mathbf{F}$}
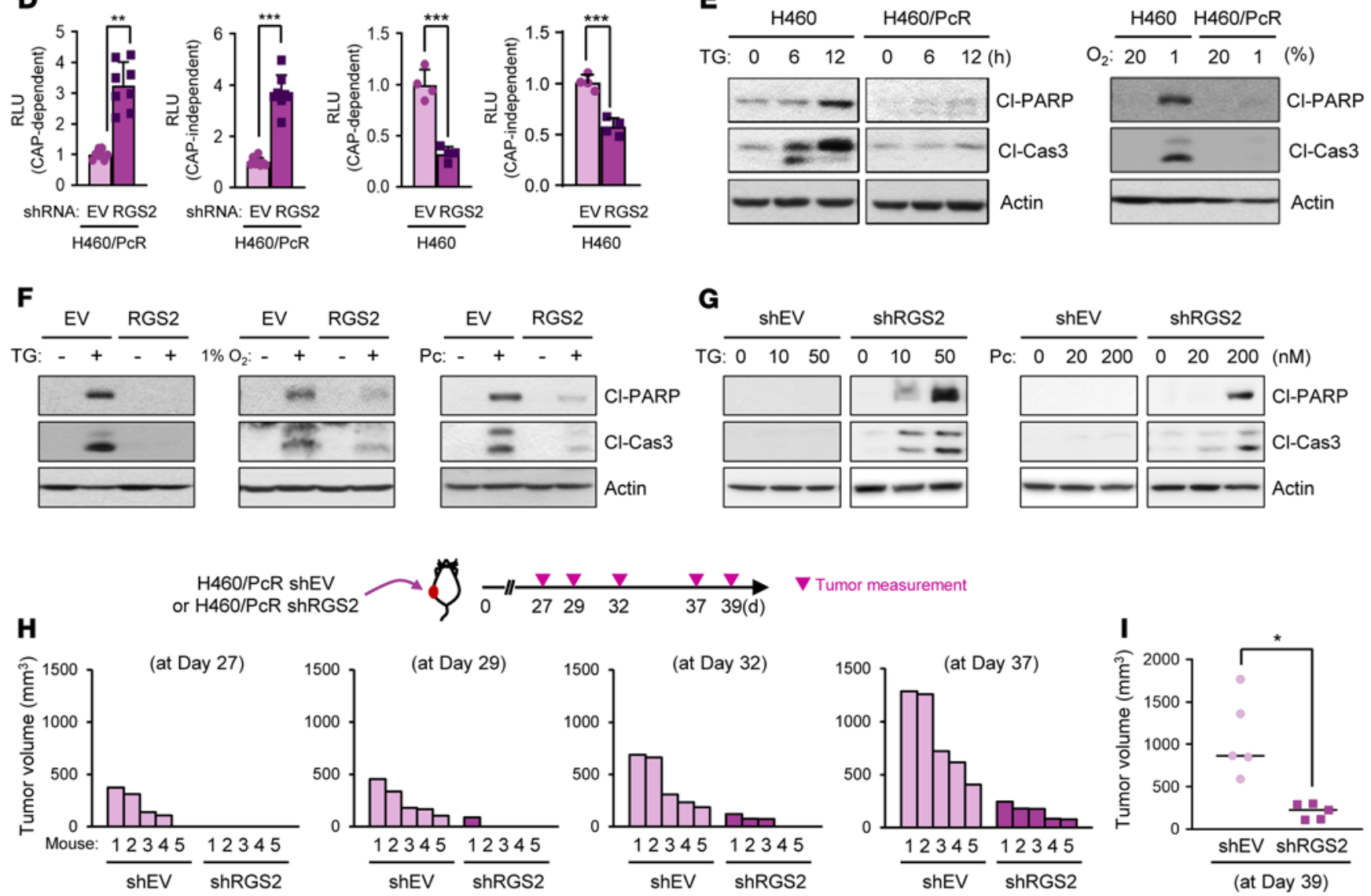

Tumor measurement
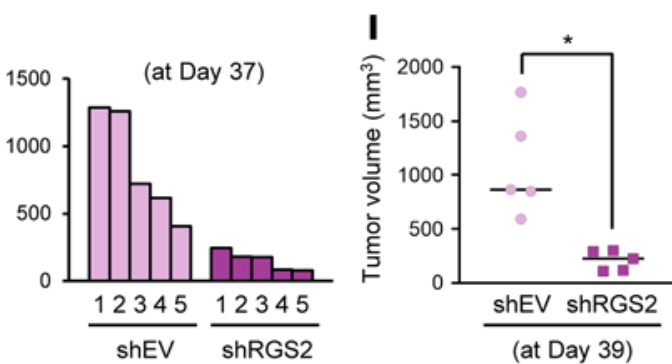

Figure 4. RGS2 is tightly associated with the acquisition of quiescence-like phenotypes in SCCs. (A) Upregulation of anchorage-dependent (A, left) and -independent colony formation (A, right) in H460/PcR cells stably transfected with RGS2 shRNA (H460/PcR-shRGS2) compared with those stably transfected with control shRNA (H460/PcR-shEV). (B-D) Modulation of the expression of cell proliferation-associated markers (B), energy metabolism (C), CAP-dependent and -independent protein translation (D) in H460/PcR-shRGS2 cells compared with H460/PcR-shEV (B-D, left) or H460 cells stably transfected with RGS2 expression vectors (H460-RGS2) compared with those stably transfected with empty vectors (H460-EV) (B-D, right). (E) ER stressinduced apoptosis caused by thapsigargin treatment (TC; $25 \mathrm{nM}$ ) or hypoxia $\left(1 \% \mathrm{O}_{2}\right)$ in $\mathrm{H} 460$ and $\mathrm{H} 460 / \mathrm{PcR}$ cells was determined by immunoblots. (F and $\left.\mathbf{G}\right)$ Modulation of UPR-induced apoptosis in H460-RGS2 (F) and H460/PcR-shRGS2 cells (G) by comparison with H460-EV and H460/PcR-shEV cells, respectively. Apoptosis was determined by immunoblots. (H and I) NOD/SCID mice implanted with H460/PcR-shRGS2 cells showed delayed tumor onset (H) and reduction in growth (I) compared with those implanted with $\mathrm{H} 460 / \mathrm{PcR}$-shEV cells ( $n=5$ per group). The data are presented as the mean \pm SD. $n=4$ for $\mathbf{A}$; $n=3$ for $\mathbf{C} ; n=4$ or 8 for $\mathbf{D}$. ${ }^{*} P<0.05,{ }^{* *} P<0.01$, and ${ }^{* * *} P<0.001$, as determined by a 2-tailed Student's $t$ test.

findings suggest that the ATF6 and IRE1 $\alpha$ branches of the UPR are activated in SCCs to ensure protein-folding homeostasis (proteostasis) in an environment with ER stresses.

Intriguingly, SCCs displayed greater levels of phospho-eIF $2 \alpha$ (peIF2 $\alpha$ ) along with downregulation of ATF4 and ATF4 target genes, including DDIT3 and PPT1R15A $(38,40)$, compared with their corresponding parental cells (Figure 5, A and B). ER stress induces PERK and eIF2 $\alpha$ phosphorylation, which is followed by preferential ATF4 translation (43). Indeed, SCCs and their respective parental cells exhibited PERK phosphorylation upon exposure to TG (Figure 5C). However, unlike their corresponding parental cells, SCCs exhibited minimal changes in phosphorylation of eIF2 $\alpha$ and protein levels of ATF4, CHOP, and GADD34 in response to the ER stressor. Consistently, exposure to chemotherapeutic drugs induced minimal effects on ATF 4 target gene expression, eIF2 $\alpha$ phosphorylation, and ATF 4 levels in the 3 SCCs (Figure 5D and Supplemental Figure 14). Thus, the upstream PERK branch appeared to be uncoupled from the downstream target genes. Based on the role of ATF4 
A
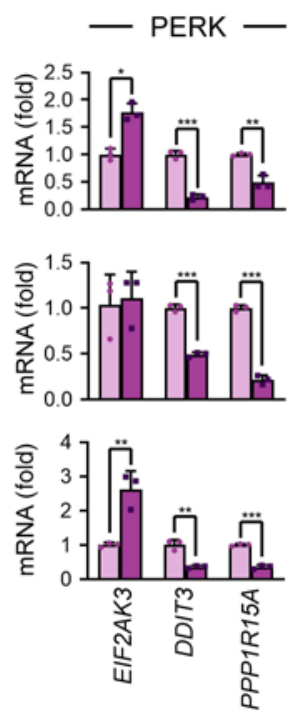

B

$\frac{\mathrm{H} 460}{\mathrm{P} \mathrm{PcR}} \frac{\mathrm{H} 1299}{\mathrm{P} \mathrm{CsR}} \frac{\mathrm{H} 1299}{\mathrm{P} \mathrm{PmR}}$

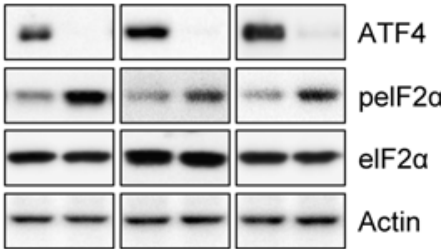

D

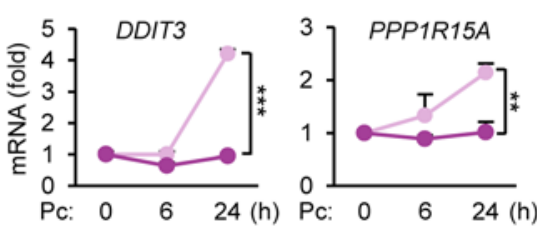

E

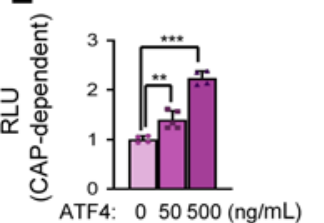

$\mathbf{F}$

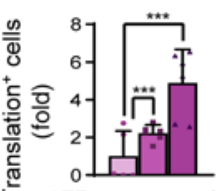

ATF4: $050500(\mathrm{ng} / \mathrm{mL})$
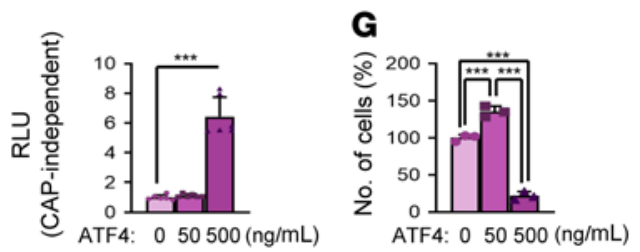

ATF6
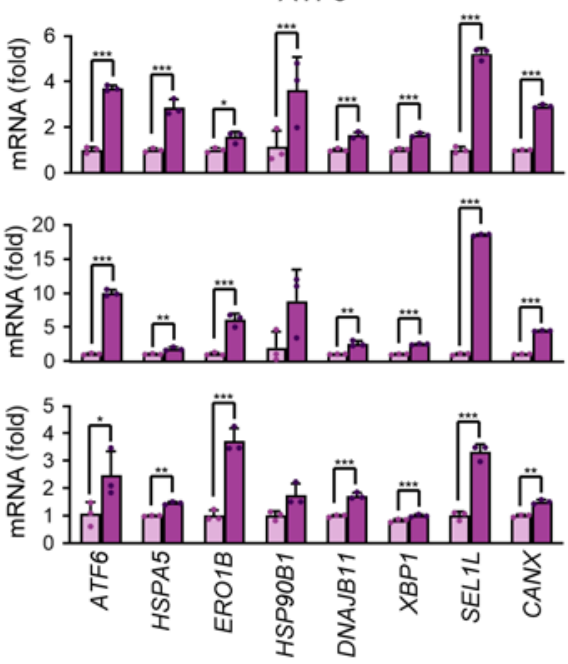

C
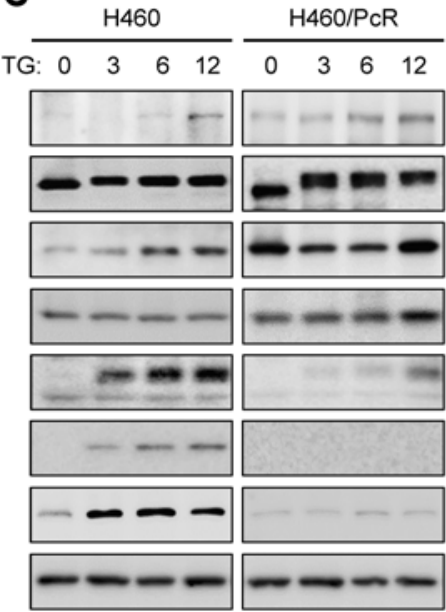

H
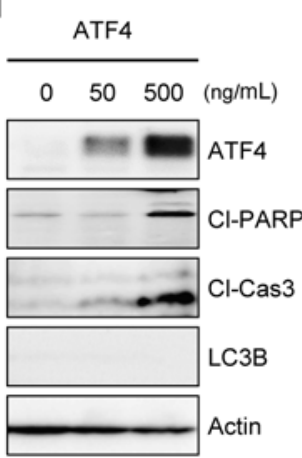
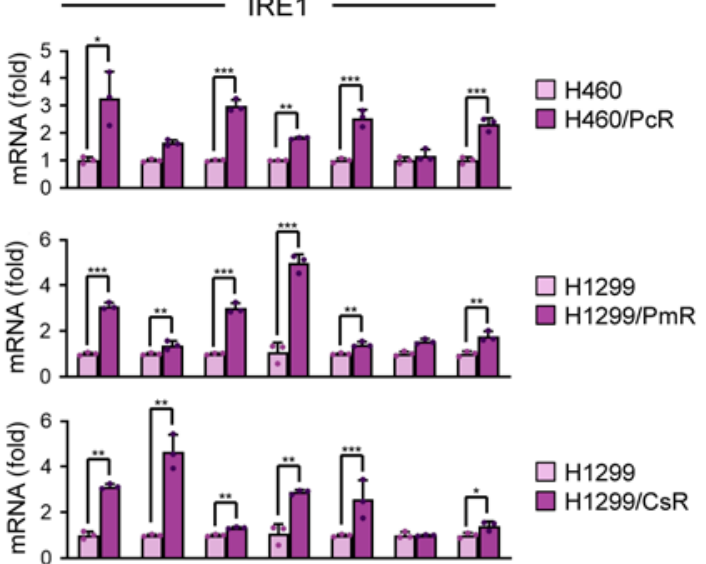

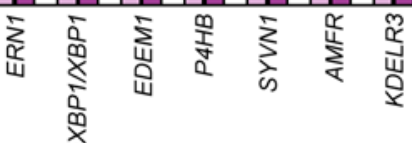

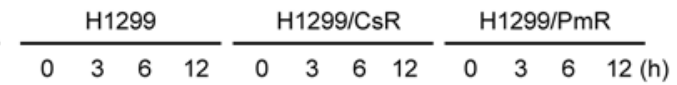

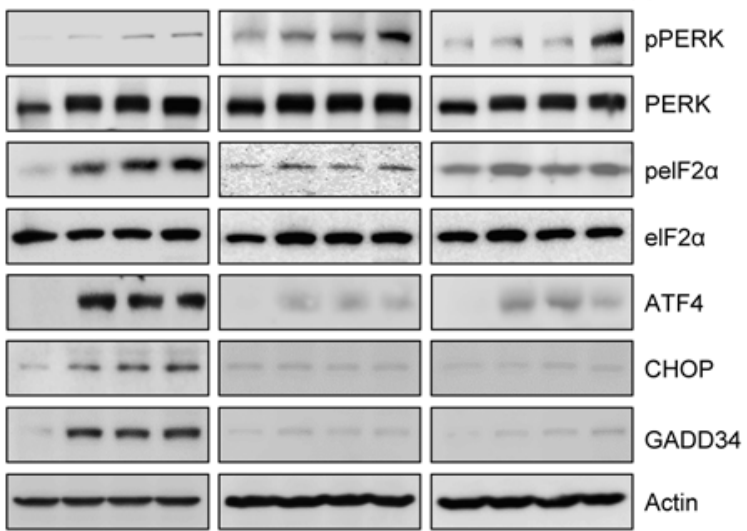

I
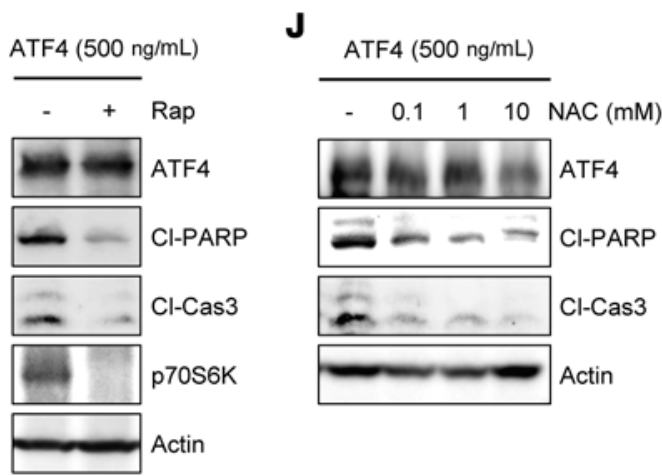

Figure 5. Reduction in ER stress-induced UPR and ATF4-mediated apoptosis in SCCs. (A) RNA expression of markers of the 3 UPR branches in SCCs by comparison with their corresponding ACCs was determined by real-time PCR. (B) Immunoblots showing changes in protein expression levels of PERK pathway components in SCCs compared with ACCs. (C) Decreased UPR induction by treatment with thapsigargin (TC; $25 \mathrm{nM}$ ) for the indicated time intervals in SCCs compared with ACCs was shown by immunoblotting. (D) Decreased ATF4 target gene induction by paclitaxel treatment (Pc; $20 \mathrm{nM}$, up to 24 hours) in $\mathrm{H} 460 / \mathrm{PcR}$ cells compared with $\mathrm{H} 460$ cells was determined by real-time PCR. (E-H) Restoration of protein translation and subsequent cell death resulting from the overexpression of ATF4. Changes in CAP-dependent and -independent protein translation (E), nascent protein synthesis $(\mathbf{F})$, cell proliferation (G) and apoptosis (H) were determined in H460/PcR cells transfected with increasing amounts of ATF4. (I and J) Attenuation of ATF4 overexpression-induced apoptosis by treatment with rapamycin (Rapa; $50 \mathrm{nM}$, for 12 hours) (I) and with the indicated concentrations of NAC for 24 hours (J). The data are presented as the mean \pm SD. $n=3$ for $\mathbf{A} ; n=3$ for $\mathbf{D} ; n=4$ or 6 for $\mathbf{E} ; n=6$ for $\mathbf{F} ; n=3$ for $\mathbf{G} .{ }^{*} P<0.05$, ${ }^{* *} P<0.01$, and ${ }^{* * *} P<0.001$, as determined by a 2-tailed Student's $t$ test (A and $\mathbf{D}$ ) and 1-way ANOVA with Dunnett's post hoc test (E-G). 
in the restoration of protein synthesis after transient suppression, induction of apoptosis, or both under ER stress $(16,17)$, we propose that a regulatory mechanism for ATF 4 expression enables SCCs to maintain survival in an environment with ER stresses.

SCCs escape ER stress-induced apoptosis by suppressing ATF4mediated protein synthesis. ATF4-mediated mRNA translation was found to induce antiapoptotic or apoptotic activities in stressed cells depending on expression levels $(18,44)$. Indeed, induced ATF4 expression caused a dose-dependent increase in mRNA translation in $\mathrm{H} 460 / \mathrm{PcR}$ cells (Figure 5, E and F; and Supplemental Figure 15). Further, restoration of ATF 4 expression to a moderate level stimulated proliferation of $\mathrm{H} 460 / \mathrm{PcR}$ cells. In contrast, overload in translation through forced ATF 4 overexpression caused a decrease in proliferation via apoptotic cell death, as shown by increases in caspase-3 and PARP cleavage (Figure 5, G and H). Moreover, ATF4-induced apoptosis was markedly attenuated in response to treatment with an mTOR inhibitor (rapamycin) (Figure 5I) or an ROS scavenger [ $\mathrm{N}$-acetyl-L-cysteine (NAC)] (Figure 5J). These findings suggest a model in which the sustained attenuation of protein synthesis, as manifested by downregulation of ATF4, endows SCCs with cellular dormancy and survival capacity during exposure to sources of ER stress.

RGS2 binds ATF4 and induces its proteasome-mediated degradation. Based on our results showing downregulation of ATF4 in RGS 2 hi SCCs and results from a previous study suggesting a modulatory link between RGS2 and ATF4 (45), we hypothesized the potential involvement of RGS2 in regulation of ATF4 expression. Indeed, H460/PcR-shRGS2 cells showed increased levels of ATF4 protein and ATF4 target gene expression along with decreased levels of eIF $2 \alpha$ phosphorylation compared with H460/PcR cells (Figure 6A). These differences in H460-RGS2 cells compared with H460 cells opposed those observed in H460/PcR-shRGS2 cells compared with H460/PcR cells (Figure 6B). These findings clearly indicated the role of RGS2 in ATF4 expression.

We then investigated the mechanism by which RGS2 regulates ATF4 expression. ATF4 transcription did not differ between SCCs and their corresponding parental cells, between H460/PcRshRGS2 and H460/PcR cells, or between H460-RGS2 and H460 cells (Figure $6 \mathrm{C}$ ). RGS2 has a regulatory role in protein synthesis (24); however, H460-RGS2 and H460 cells showed a similar rate of de novo synthesis of the ATF4 protein (Figure 6D). Intriguingly, the half-life of the ATF4 protein was significantly shorter in RGS2-transfected H460 and H1299 cells than in their corresponding control cells (Figure 6E). Moreover, pretreatment with MG132 increased the levels of polyubiquitinated forms of ATF4 in H460/ PcR cells and RGS2-transfected H460 cells compared with control cells (Figure 6F). By contrast, shRNA-mediated depletion of RGS2 suppressed ATF 4 polyubiquitination in H460/PcR cells. Coimmunoprecipitation assays using $\mathrm{H} 460 / \mathrm{PcR}$ cells in which proteasome machinery was inactivated by MG132 treatment showed an association between ATF 4 and RGS2 (Figure 6G). H460 cells, in which RGS2 expression was enforced by transfection before MG132 treatment, confirmed RGS2 and ATF4 association (Figure 6H). The input lanes showed that MG132 proteasome inhibitor treatment restored ATF4 expression in RGS2-transfected H460 cells.

To identify the active domains within RGS2 that confer ATF4-binding capacity, we generated bacterial expression vectors carrying full-length (FL) RGS2 and 3 deletion mutants in which parts of the carboxyl terminus ( $\triangle \mathrm{C} 79$ and $\Delta \mathrm{C} 169)$ or residues upstream of RGS2 ( $\triangle$ N79) were deleted (Figure 6I). A pulldown assay using bacterial proteins revealed that both FL RGS2 and 2 RGS2 mutants ( $\triangle \mathrm{C} 79$ and $\triangle \mathrm{C} 169)$ associated with ATF4 in H460 cell lysates. By contrast, the truncation mutant without the residues upstream of RGS2 ( $\triangle \mathrm{N} 79$ ) failed to associate with ATF4 (Figure 6J). Moreover, protein translation was prominently suppressed in FL RGS2-transfected or $\Delta$ C79-transfected $\mathrm{H} 460$ cells compared with empty vector-transfected (EV-transfected) or $\Delta$ N79-transfected cells (Figure 6K). These results suggest that an interaction between RGS2 and ATF4 via the RGS2 N-terminal residues (1-79) mediated ATF4 proteasomal degradation, leading to the prolonged suppression of global mRNA translation in NSCLC SCCs.

$R G S 2^{h i} / A T F 4^{l o}$ SCCs are present in NSCLCPDXs and are enriched during chemotherapy. Our data thus far have shown that SCCs are characterized by high RGS2 and low ATF4 expression (RGS2hi $\mathrm{ATF} 4^{\mathrm{lo}}$ ) and that they emerge to escape ER stress. We thus determined whether RGS2 ${ }^{\text {hi }} / \mathrm{ATF} 4^{\text {lo }}$ SCCs emerge within residual lung tumors during chemotherapy in vivo. As shown by Western blotting and IHC analyses, residual $\mathrm{H} 460$ xenograft tumors in NOD/ SCID mice and Lewis lung carcinoma (LLC) allograft tumors in C57BL/ 6 mice treated with 3 cycles of a clinically relevant combinatorial chemotherapeutic regimen (i.e., a 7-day regimen comprising Pc/Cs treatment for 1 day followed by a 6-day drug holiday) displayed markedly increased RGS2 and decreased ATF4 protein levels compared with the corresponding vehicle-treated control tumors (Figure 7, A-H; and Supplemental Figure 16A).

To validate the clinical relevance of these results, we assessed whether RGS2 ${ }^{\text {hi }} / \mathrm{ATF}^{\text {lo }}$ drug-tolerant SCCs are present in human primary NSCLC tumors and selected for during chemotherapy (Figure 7I). Because of technical challenges to sampling residual human lung tumor tissues, we monitored $\mathrm{RGS} 2^{\text {hi }} / \mathrm{ATF} 4^{\text {lo }}$ populations in 3 different NSCLC PDX tumors before and after chemotherapy. Consistent with the results in the analysis of an NSCLC TMA (Figure $2 G$ ), an inverse correlation between RGS2 and Ki67 expression was obtained in the PDX models (Figure 7J). During the administration of 3 cycles of the clinically relevant combinatorial chemotherapeutic regimen, the PDX tumors shrank to less than $50 \%$ of their original volume within 22 days (Figure $7 \mathrm{~K}$ ). Although $\mathrm{ATF}^{+}$cells were detectable in drug-naive tumors at baseline by IHC analysis, they were decreased in number (2.5-fold on average) during chemotherapy. Conversely, we observed a time-dependent increase in RGS2 ${ }^{+}$cells (5-fold on average) in residual tumors compared with their baseline tumors during chemotherapy (Figure 7L and Supplemental Figure 16B). These data suggest that distinct RGS2 ${ }^{\text {hi }} / \mathrm{ATF} 4^{\text {lo }}$ subpopulations within residual tumor lesions are selected during chemotherapy.

RGS2 depletion induces apoptosis in RGS2 $2^{\text {hi }} / A T F 4^{l o}$ SCCs and prevents tumor relapse. To link these findings to clinical application, we evaluated the effects of RGS2 depletion on tumor relapse after chemotherapy. $\mathrm{H} 460$ xenograft tumors that apparently regressed during chemotherapy resumed growth after the cessation of chemotherapy (Figure 8A). RGS2 ablation by injection with a liposome-encapsulated siRNA significantly suppressed the outgrowth of residual xenograft tumors by inducing apoptosis, as evidenced by IHC analysis of cleaved caspase-3 levels in the tumors (Figure 8, B and C). The siRNA-mediated RGS2 ablation also suppressed H460/PcR-mediated xenograft tumor growth (Figure 8D and Supplemental Figure 17A). 

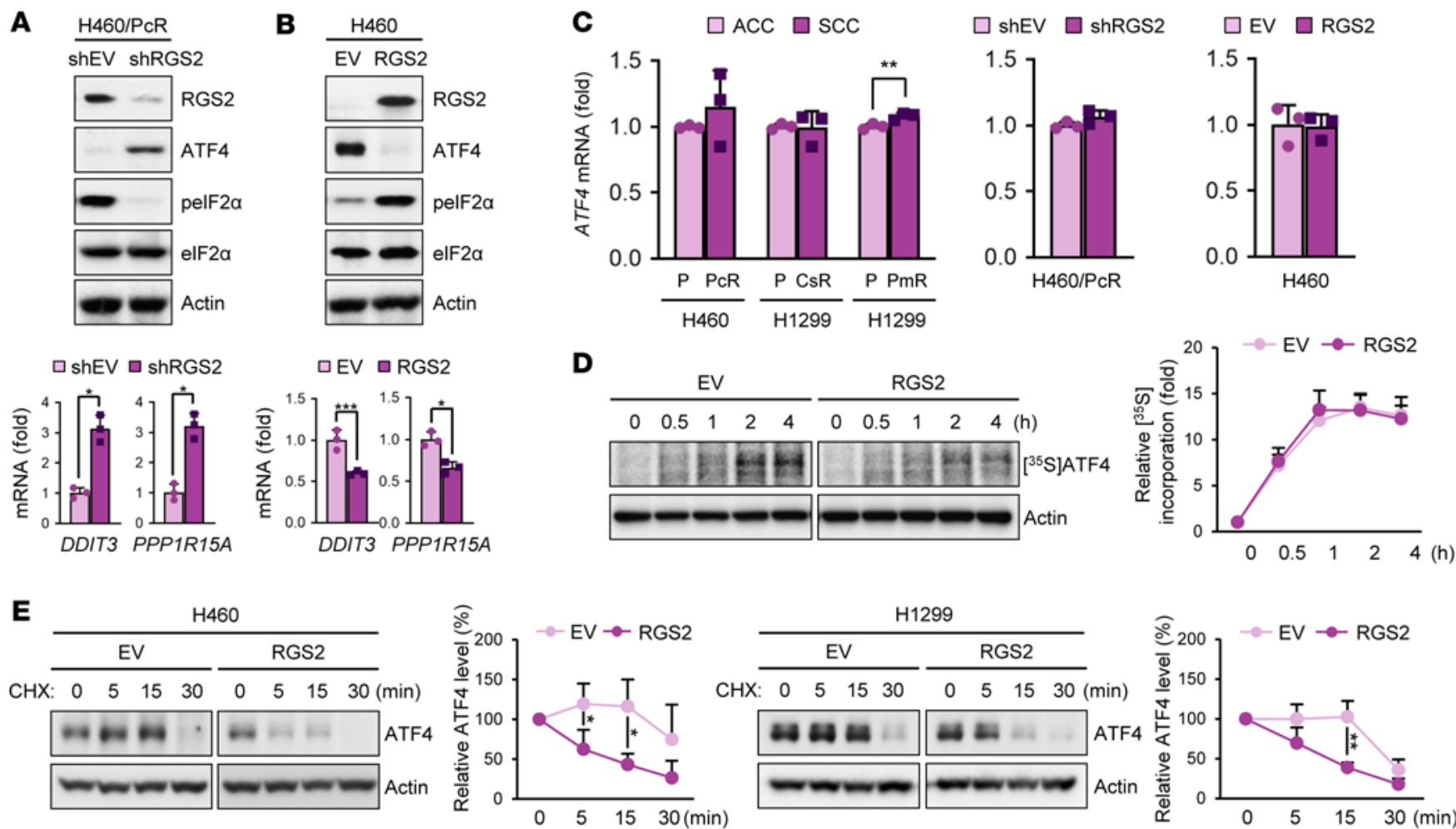

$\mathbf{F}$
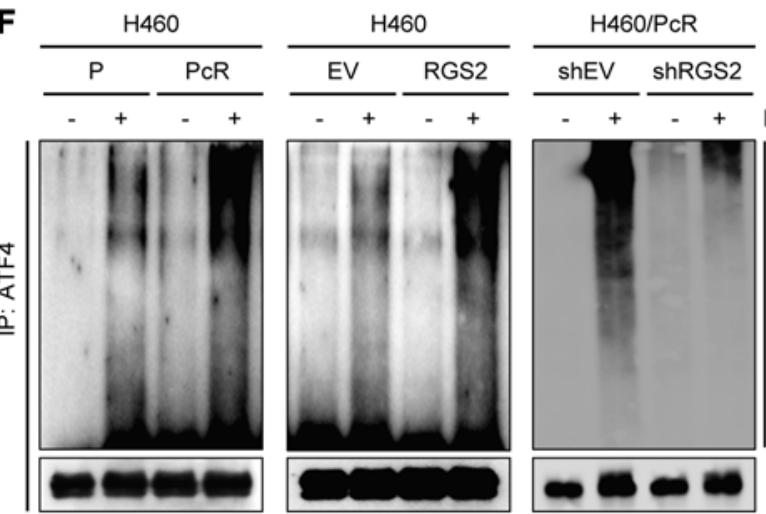

G

$\mathrm{H} 460 / \mathrm{PcR}$

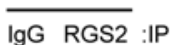

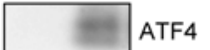

IB: Ub ATF4-(Ub)

IB: ATF4

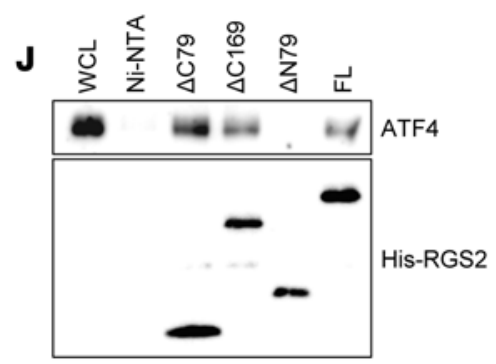

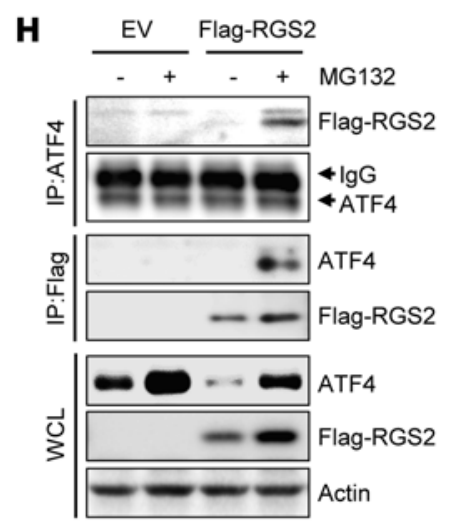

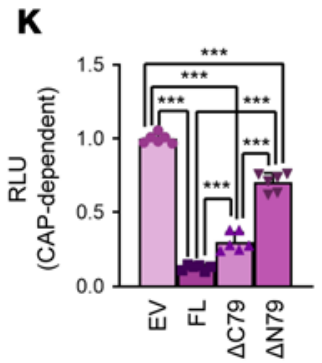


Figure 6. Regulation of ATF4 protein stability by RGS2 via interaction in SCCs. (A and B) Changes in the expression of ATF4, phosphorylated elF2 $\alpha$, and ATF4 target genes in H460/PcR-shRGS2 compared with H460/PcR-shEV (A) and in H460-RGS2 compared with H460-EV (B), as determined by immunoblotting and real-time PCR. (C) ATF4 mRNA expression in SCCs compared with the corresponding ACCs, in $\mathrm{H} 460$ /PcR-shRGS2 compared with $\mathrm{H} 460$ / PcR-shEV and in H460-RGS2 compared with H460-EV was analyzed by realtime PCR. (D) Regulation of de novo ATF4 protein synthesis in H460-EV and H460-RGS2 cells was determined by autoradiography using ${ }^{35}$ S-labeled ATF4 immunoprecipitants. Immunoblotting result is representative of triplicates. (E) Changes in ATF4 protein stability regulated by RGS2 were determined by immunoblotting using lysates from cells treated with cycloheximide $(\mathrm{CHX} ; 100 \mu \mathrm{g} / \mathrm{mL}$ ) for the indicated time periods. Immunoblotting result is representative of triplicates. (F) ATF4 ubiquitination in SCCs compared with ACCs and in cells with modulated RCS2 expression was determined by immunoprecipitation of ATF4 and subsequent immunoblotting for ubiquitin. (C) The endogenous interaction between RGS2 and ATF4 was determined by coimmunoprecipitation assay in H460/PcR cells treated with MC132 (10 $\mu \mathrm{M}$ for 4 hours). (H) The interaction between ectopic RCS2 and endogenous ATF4 was determined by coimmunoprecipitation assay in $\mathrm{H} 460$ cells treated with or without MG132 (10 $\mu \mathrm{M}, 4$ hours). (I) Diagrams showing the full-length (FL) and mutant recombinant RCS2 proteins. (J) The interaction between ATF4 and FL or mutant RCS2 proteins was determined by pulldown assay. (K) Regulation of protein translation by overexpression of FL or mutant RGS2 proteins. The data are presented as the mean \pm SD. $n=3$ for $\mathbf{A}-\mathbf{E} ; n=6$ for $\mathbf{K} .{ }^{*} P<0.05$, ${ }^{* *} P<$ 0.01 , and ${ }^{* *} P<0.001$, as determined by a 2-tailed Student's $t$ test $(\mathbf{A}-\mathbf{E})$ and 1-way ANOVA with Dunnett's post hoc test (K).

We also observed enhanced antitumor activities after combined treatment with RGS2 siRNA and Pc (Figure 8E and Supplemental Figure 17B). Similar results were obtained with 2 different PDX models (Figure 8, F and G; and Supplemental Figure 17C). These findings suggest the potential of targeting RGS2 to suppress SCC-derived tumor relapse and to restore chemosensitivity.

Abrogation of translational control sensitizes SCCs to ER stressinduced apoptosis and facilitates the eradication of SCCs in vitro and in vivo in combination with chemotherapy. Because anticancer drugs that target RGS2 are currently unavailable, we searched the FDAapproved drugs list (1,622 compounds) for clinically available therapeutics that can restore translational programs. We focused on PDE5 inhibitors (sildenafil and tadalafil) that can activate protein synthesis in skeletal muscle $(46,47)$. Indeed, these inhibitors induced a dose-dependent increase in protein synthesis (Supplemental Figure 18A) and suppressed the characteristics of quiescence, e.g., upregulated p38 phosphorylation, increases in the expression of p21 and p27, and decreases in ERK phosphorylation and cyclin D1 expression, in SCCs (Figure 9A). Notably, in comparison with PDE5 inhibitor treatment alone, combined treatment with the PDE5 inhibitor and Pc markedly suppressed the viability and colony-forming capacity of H460/PcR cells by inducing apoptosis (Figure 9, B-D). Consistently, combined treatment with Pc and dibutyryl-cyclic GMP (db-cGMP), a cGMP analog that acts similar to PDE5 inhibitors, exhibited greater inhibition of the colony-forming ability of H460/PcR cells compared with db-cGMP treatment alone (Supplemental Figure 18B). Consistent with the effects of cGMP signaling on the insulin signaling pathway $(48,49)$, these inhibitors increased Akt, mTOR, and $4 \mathrm{E}-\mathrm{BP} 1$ phosphorylation (Figure 9A), suggesting that the effects of these drugs on protein synthesis are mediated by PI3K/Akt/mTOR signaling and subsequent 4E-BP1 phosphorylation. Indeed, apoptosis was markedly attenuated by treatment with rapamycin (Figure
9E) or NAC (Figure 9F), confirming that increased protein synthesis and ROS production are key drivers of apoptotic death in sildenafil and Pc treated H460/PcR cells.

We evaluated the efficacy of sildenafil treatment in combination with chemotherapy in vivo. Because the results of treatment with both PDE5 inhibitors in vitro were similar, we used only sildenafil for this in vivo study. We started the drug treatment in mice within 1 week of NSCLC cell injection to assess the effects of the combined treatment on outgrowth of SCC in vivo. Compared with single treatments, combination treatment with sildenafil and chemotherapy significantly suppressed the outgrowth from H460/PcR and H1299/CsR xenograft tumors (Figure 9G) and PDX tumors (Figure 9H). IHC analysis of the tumors revealed that compared with the other treatments, the combination treatment induced a statistically significant increase in caspase-3 cleavage (20-fold increase on average compared with the vehicle-treated control) (Figure 9I and Supplemental Figure 18C). We next evaluated the efficacy of the combined treatment in mice carrying H460/PcR xenograft tumors of approximately $200 \mathrm{~mm}^{3}$ in volume. Compared with the single treatments, the combination treatment led to significant growth inhibition of the established tumors (Supplemental Figure 18D). The combination therapy did not result in weight loss in excess of that seen with inhibitor monotherapy or vehicle treatment (Supplemental Figure 18E). These results suggest that restoring protein synthesis by PDE5 inhibitors imposes additional ER stress on SCCs, thus facilitating their eradication by chemotherapy and ultimately preventing tumor relapse in vivo.

\section{Discussion}

Preventing chemoresistance and tumor progression through the identification and characterization of SCCs, understanding their biology, and the development of novel strategies to target SCCs are emerging as logical salvage approaches for chemotherapy (50). We showed herein a rare population of RGS2 $2^{\text {hi }} / A T F 4^{\text {lo }}$ SCCs in NSCLC in which the 3 key ER stress signaling branches were tailored to maintain a stable, quiescence-like phenotype and ensure survival under conditions of chronic microenvironmental ER stresses. In ACCs, the IRE1 $\alpha$, PERK, and ATF6 branches of the UPR are activated upon exposure to various sources of ER stress, including chemotherapy $(16,17)$. By our model, PERK-induced phosphorylation of eIF $2 \alpha$ attenuated global mRNA translation; however, selective translation of mRNA encoding ATF4, a transcription factor for GADD34, led to the restoration of protein synthesis (Figure 10, left). The release of active ATF6 through proteolytic processing and production of sXBP1 through IRE1 $\alpha$-mediated ribonuclease activity mediated the restoration of ER homeostasis by inducing transcriptional regulation of genes involved in protein folding or ER-associated degradation (ERAD). We hypothesize that SCCs survive in hostile microenvironments with sustained ER stress partially by activating ATF6-mediated and IRE1 $\alpha$-mediated protein folding and ERAD (Figure 10, middle). Our results specifically emphasize a unique mechanism in SCCs wherein RGS2 overexpression ensured prolonged translational arrest by binding ATF4 and inducing its proteasome-mediated degradation. Finally, we have shown that disturbance of proteostasis by genetic ablation of RGS2 or pharmacological disruption of translational control rendered SCCs sensitive to ER stress-induced apoptosis, which ultimately restored chemosensitivity and suppressed tumor 
A

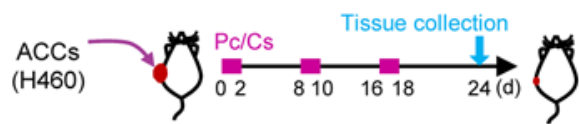

B

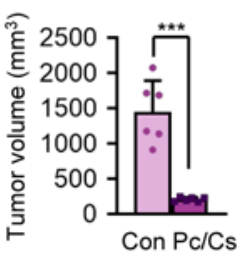

C

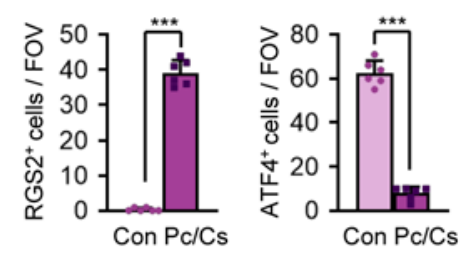

D

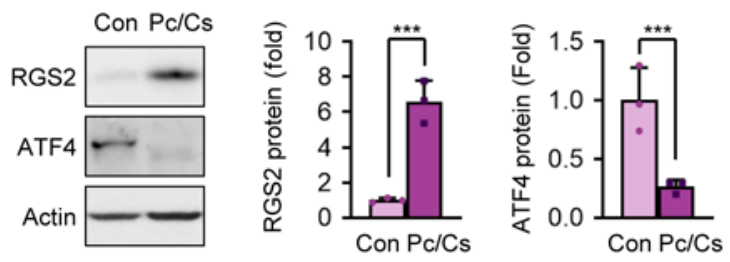

E

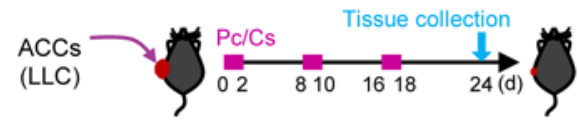

$\mathbf{F}$

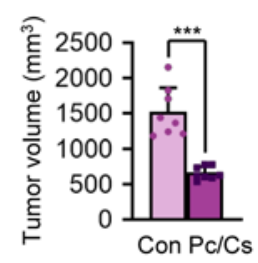

G

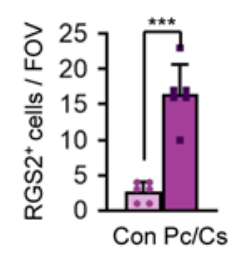

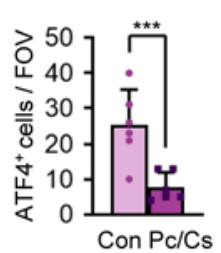

H

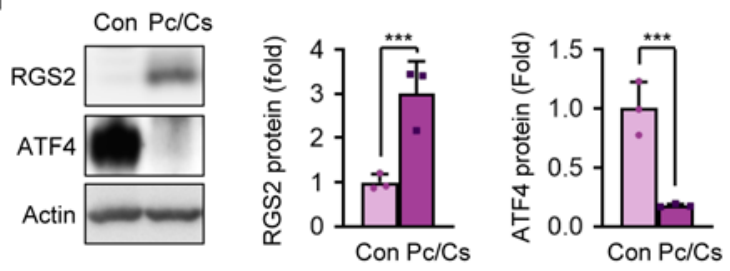

I

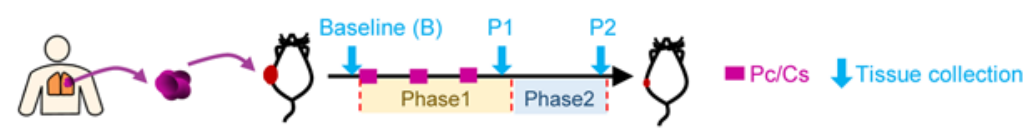

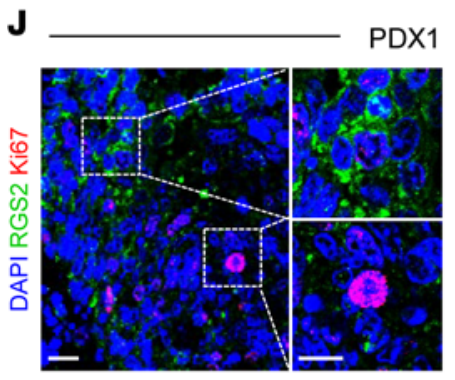
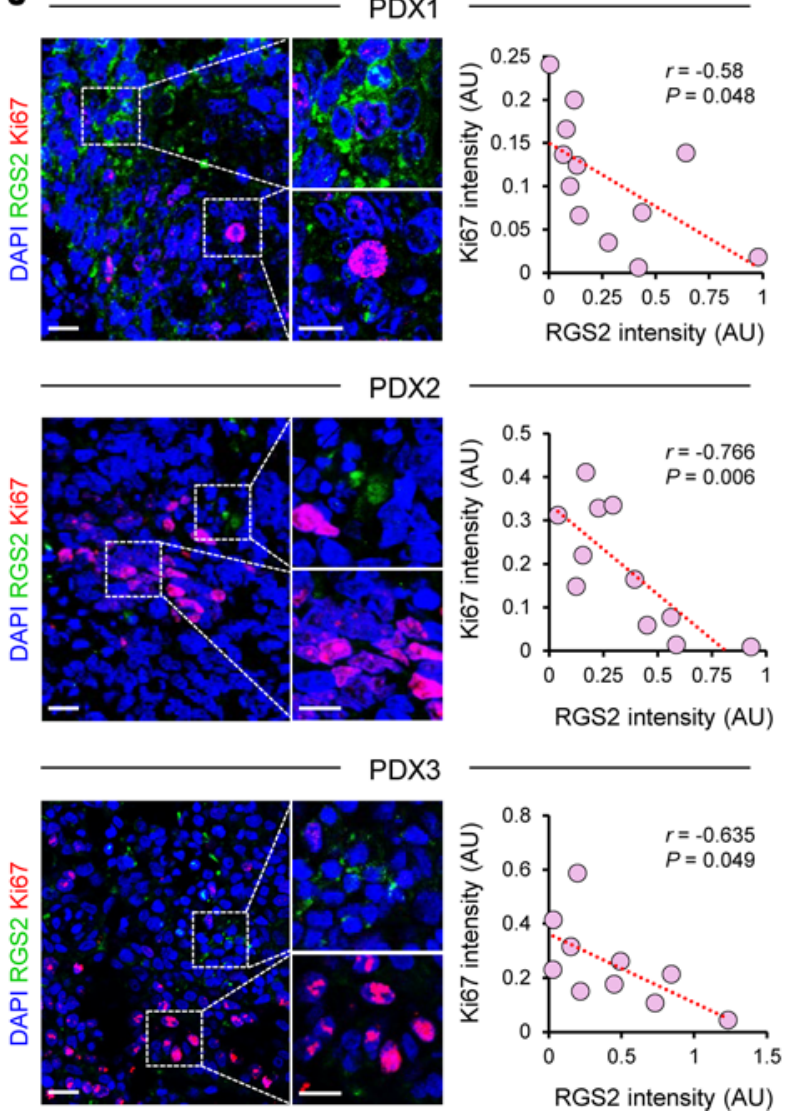

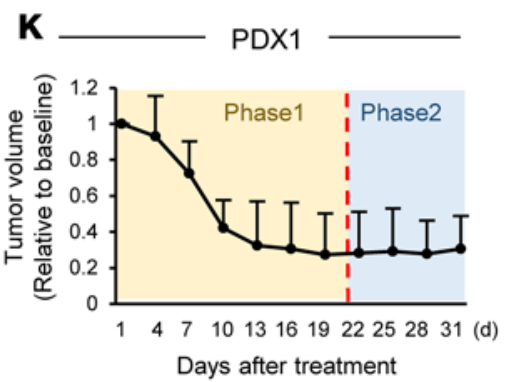

$\mathbf{L}$
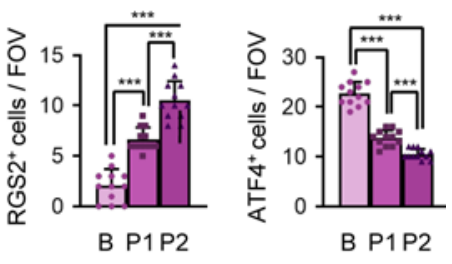

PDX2

PDX2
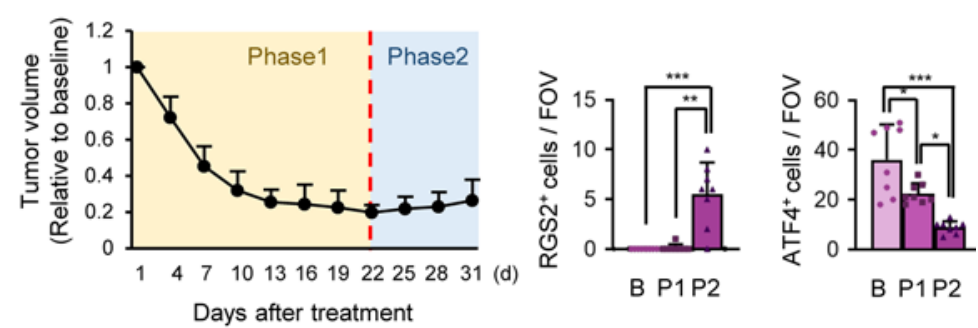

PDX3

PDX3
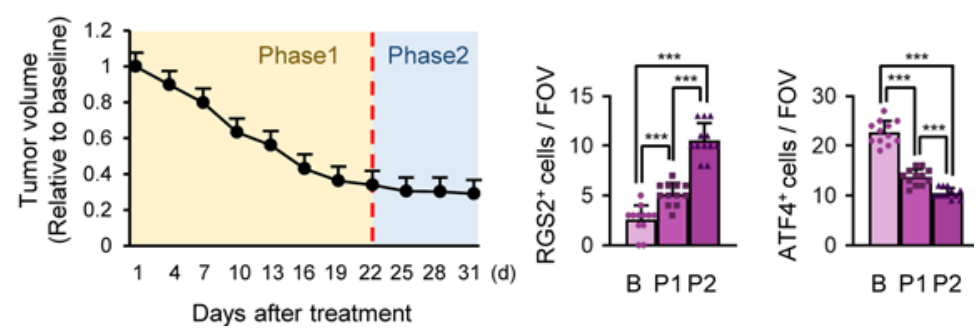
Figure 7. Enrichment of RCS2 ${ }^{\text {hi }} / A T F 4^{10}$ SCCs in chemoresistant tumors. (A-D) H460 xenografts in NOD/SCID were subjected to 3 cycles of vehicle or paclitaxel/cisplatin ( $\mathrm{Pc} / \mathrm{Cs}$ ) treatment ( $n=6$ per group) (A). (B) Tumor growth of vehicle- or Pc/Cs-treated $\mathrm{H} 460$ xenograft tumors. (C) IHC analyses showing the expression of RCS2 and ATF4 in the vehicle- or Pc/Cs-treated H460 xenograft tumors. (D) Protein expression level of RGS2 and ATF4 in the vehicle- or $\mathrm{Pc} / \mathrm{Cs}$-treated $\mathrm{H} 460$ xenograft tumors. Representative immunoblotting is shown. (E-H) LLC allografts in C57BL/6 mice were subjected to 3 cycles of vehicle or $\mathrm{Pc} / \mathrm{Cs}$ treatment ( $n=8$ per group) (E). (F) Tumor growth of vehicle- or Pc/Cs-treated LLC allograft tumors. (C) IHC analyses showing expression of RGS2 and ATF4 in the vehicle- or Pc/Cs-treated LLC allograft tumors. (H) Protein expression level of RGS2 and ATF4 in the vehicle- or Pc/ Cs-treated LLC allograft tumors. Representative immunoblotting is shown. (I-L) PDX tumors in NOD/SCID mice were subjected to the 3 cycles of Pc/ Cs treatment. At 3 different time points, PDX tumors were harvested and analyzed. ( $n=5$ per each phase) (I). (J) Correlation analysis between RGS2 and Ki67 expression in PDX tumors. Scale bar: $50 \mu \mathrm{m}$, inset: $20 \mu \mathrm{m}$. (K) Changes in the relative tumor volume after $\mathrm{Pc} / \mathrm{Cs}$ treatment. (L) Intratumoral expression of RGS2 and ATF4 in each phase of Pc/Cs-treated tumors. The data are presented as the mean \pm SD. $n=6$ for $\mathbf{C}, \mathbf{G} ; n=3$ for $\mathbf{D}, \mathbf{H} .{ }^{*} P<0.05$, ${ }^{*} P<0.01$, and ${ }^{* *} P<0.001$, as determined by a 2-tailed Student's $t$ test (A-E) and 1-way ANOVA with Dunnett's post hoc test (K).

relapse (Figure 10, right). This finding suggests that translational arrest has a pivotal role in SCC survival in hostile environments.

The molecular mechanisms underlying cancer cell dormancy and dormancy-mediated drug resistance have long been enigmatic. Our data from RNA-Seq analyses and validation studies in 5 different CSFE ${ }^{\text {hi }}$ SCC populations from NSCLC cell lines and PDX tumors versus their corresponding $\mathrm{CSFE}^{\mathrm{lo}}$ populations showed that NSCLC SCCs are characterized by transcriptional upregulation of 11 genes involved in the regulation of cell proliferation, including RGS2. Importantly, our inquiry of publicly available data sets and IF analysis of NSCLC TMA provided evidence that supported the role of RGS2 as a regulator of cancer cell dormancy and response to cellular stresses and a marker of poor prognosis in NSCLC. The function of RGS2 as a stress response element has been extensively described (20-22). RGS2 has been also implicated in other types of cancers. For example, RGS2 expression was associated with poor prognosis in advanced prostate cancer (51); downregulation of RGS2 was observed during prostate cancer progression (51); and the role of RGS2 in regulating the proangiogenic function of myeloid-derived suppressor cells (MDSCs) in tumors was demonstrated (52). Hence, although an implication of the other significantly upregulated genes has not been ruled out, the functional features of the SCCs in $\mathrm{CSFE}^{\mathrm{hi}}$ subpopulations can be due at least in part to RGS2 expression.

To assess whether RGS2 functions as a driver of SCC-mediated tumor relapse, we attempted to establish a preclinical model of RGS2 ${ }^{\text {hi }}$ SCCs. Because sampling functionally defined RGS2 ${ }^{\text {hi }}$ SCCs from patients is currently impossible, we adapted chemotherapeutic drugs that target ACCs to NSCLC cell lines and PDX tumors. We obtained 2 groups of surviving subpopulations with distinct proliferation states: one group harboring a similar level of RGS2 expression and proliferation rate compared with their corresponding parental cells; another group harboring increased levels of RGS2 expression along with representative quiescence-like traits; resistance to apoptosis induced by ER stresses, including chemotherapy; and delayed tumor initiation. Upon siRNA-mediated loss of RGS2 expression,
RGS2 ${ }^{\text {hi }}$ SCCs recovered their proliferative activities, but underwent apoptosis in environments with ER stressors both in vitro and in vivo. Conversely, NSCLC ACCs exhibited attenuated proliferation but became resistant to ER stress-induced apoptosis upon the forced overexpression of RGS2. These findings defined RGS2 as a major player in the acquisition of functional features of SCCs. The RGS2 ${ }^{\text {hi }}$ SCCs might arise by a de novo transition of ACCs through specific genetic alterations or transcriptional reprogramming during exposure to chemotherapy. However, we observed RGS2 ${ }^{\text {hi }}$ SCCs within NSCLC cell lines, PDX tumors, and NSCLC tissues from patients before the onset of chemotherapy. Given the resemblance between SCCs and CSCs (53), a causative link between CSCs and SCCs can be inferred. The established RGS2 ${ }^{\text {hi }}$ SCCs in our study, however, did not show a CSC-like phenotype, suggesting that stemness, dormancy, and drug resistance are not always linked, at least in NSCLC. Therefore, RGS2 ${ }^{\text {hi }}$ SCCs might represent an authentically distinct NSCLC subpopulation harboring unique biology and characteristics.

An important question is how RGS2 endows SCCs with the quiescence and survival capacities. Our data showed that RGS 2 hi SCCs acquired IRE1 $\alpha$-mediated and ATF6-mediated protein folding and ERAD, suggesting that proteostasis through activation of the UPR plays a major role in the functional features of SCCs. Of note, RGS $2^{\text {hi }}$ SCCs have persistent eIF $2 \alpha$ phosphorylation but reduced levels of the ATF 4 protein and its target gene expressions. UPR activation by PERK-mediated eIF2 $\alpha$ phosphorylation in environments with ER stressors generally diminishes the translation of most mRNAs (54). Subsequently, selective translation of ATF4 is uninterrupted or even augmented through particular alternate pathways $(16,17,55)$, which in turn mediates the transcription of UPR-responsive genes to restore protein synthesis $(56,57)$. Increased protein synthesis under conditions of sustained ER stress may, however, augment the unfolded protein load, which constitutes a signal for apoptosis. Indeed, in some contexts, ATF4-mediated protein synthesis results in ER stress-induced apoptotic cell death by generating ROS (18). Consistent with these observations, we found that RGS2 $2^{\text {hi }}$ SCCs with forced ATF 4 overexpression seemed to undergo apoptotic cell death mediated by robust mRNA translation and ROS production. We further discovered a unique mechanism wherein RGS2 binds the ATF4 protein and induces its proteasome-mediated degradation in SCCs. Thus, our findings imply that RGS2-mediated regulation of ATF 4 expression ensured translational arrest and survival capacity in SCCs under environments with ER stressors, which allowed SCCs time to remodel the microenvironment to support their proliferation. Indeed, an RGS2-targeting genomic approach markedly suppressed tumor relapse from residual tumors of NSCLC xenografts and PDXs upon completion of chemotherapy.

RGS2 expression tends to be increased in response to cellular stresses, similar to those that inhibit protein synthesis $(20,22,58)$. RGS2 was proposed to regulate protein synthesis through its ability to interfere with the eIF2-eIF2B $\varepsilon$ interaction and the eIF2-eIF2B GTPase cycle (24), which require the eIF2B $\varepsilon$-binding domain (amino acids 79-116) and the established RGS domain (amino acids 84-197), respectively. We found that RGS2-mediated ATF4 degradation mapped to the $\mathrm{N}$-terminal amino acid residues $1-79$. Hence, we think that RGS2 likely regulates the rate-limiting initiation stage of protein synthesis through 3 independent but comparable mechanisms. A recent study demonstrates the positive regulation of ATF 4 

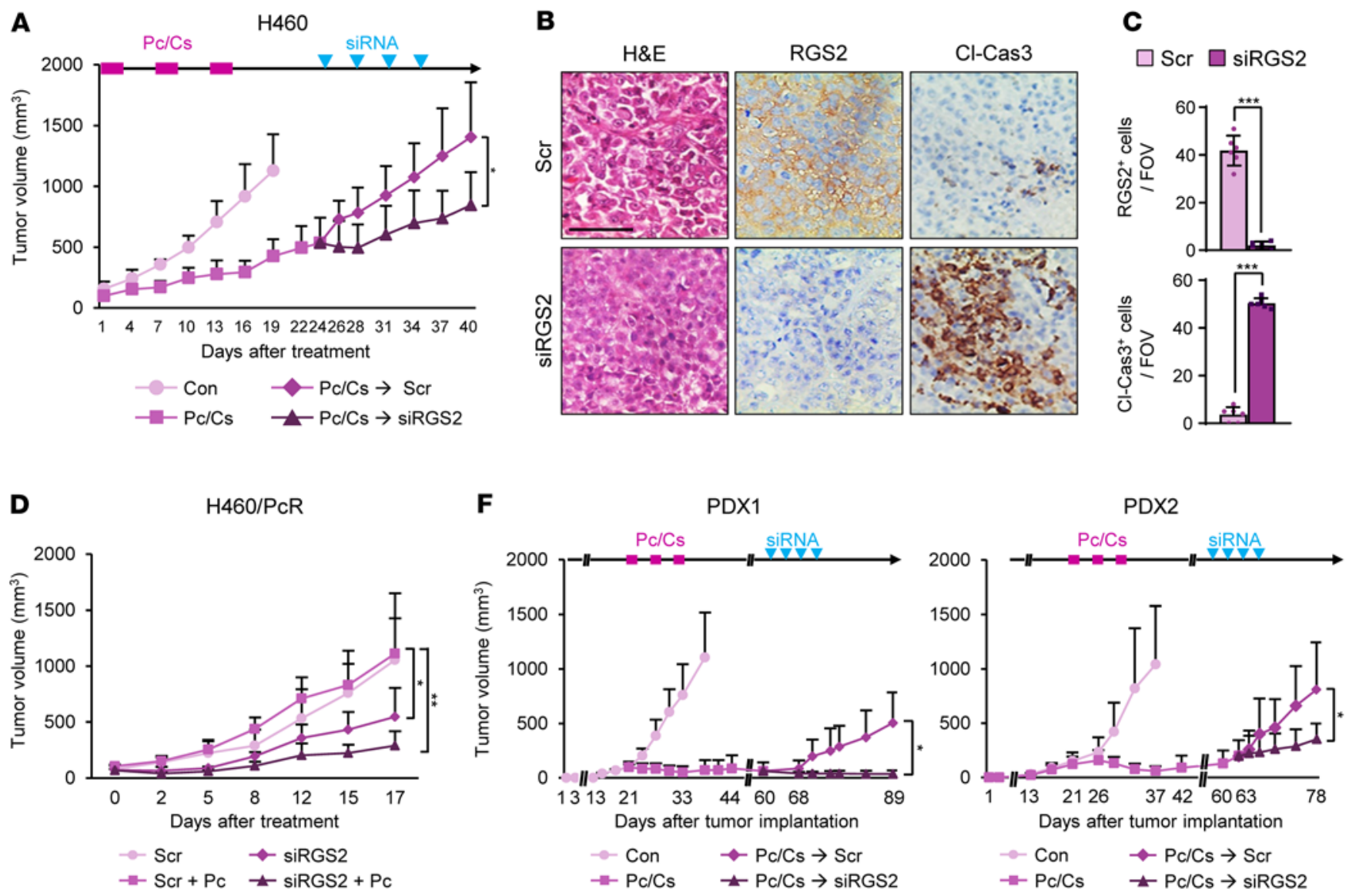

\section{E $\quad \square$ Scr $\square \operatorname{siRGS2}$}

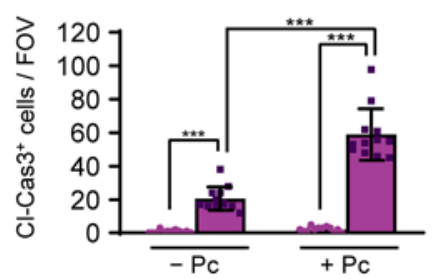

G

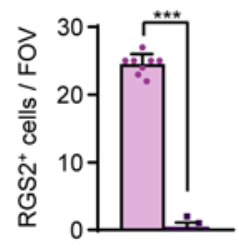

$\square$ Scr $\square$ siRGS2

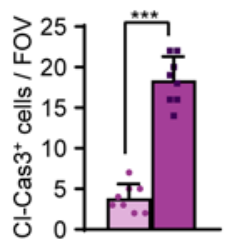

$\square$ Scr

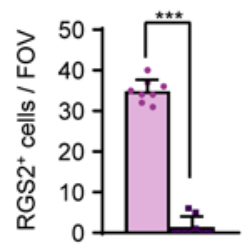

\section{siRGS2}

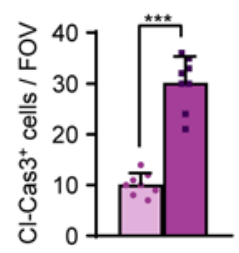

Figure 8. Prevention of tumor relapse by depletion of RGS2 ${ }^{\text {hi }} /$ ATF $^{10}$ SCCs enriched during chemotherapy. (A-C) NOD/SCID mice bearing H460 xenograft tumors were subjected to 3 cycles of a Pc/Cs treatment. After treatment, relapsed tumors were intratumorally injected with scrambled or RGS2 siRNAs ( $n$ $=6$ per group). (A) Changes in tumor growth in each group. (B) Representative IHC results for RGS2 and cleaved caspase-3 (CI-Cas3) expression in tumors harvested at the end of the experiment. Scale bar: $50 \mu \mathrm{m}$. (C) IHC analyses for RGS2 and Cl-Cas3 in scrambled or RGS2 siRNA-treated relapsed tumors. (D and E) H460/PcR xenograft tumors in NOD/SCID mice were treated with either RGS2 siRNAs alone or in combination with paclitaxel. (D) Changes in H460/ PcR xenograft tumor volume in each group. (E) IHC analyses for Cl-Cas3 in tumors at the end of combinational treatment. (F and G) Two PDX tumors were implanted in the NOD/SCID mice and subjected to 3 cycles of vehicle or Pc/Cs treatment. Relapsed tumors were intratumorally injected with scrambled or RGS2 siRNA. (F) Changes in tumor growth in each group. (C) IHC analyses for RGS2 and Cl-Cas3 in tumors harvested at the end of the experiment. The data are presented as the mean \pm SD. $n=6$ for $\mathbf{C} ; n=12$ for $\mathbf{E} ; n=8$ for $\mathbf{G} .{ }^{*} P<0.05,{ }^{* *} P<0.01$, and ${ }^{* * *} P<0.001$, as determined by 2 -tailed Student's $t$ test $(\mathbf{A}, \mathbf{C}, \mathbf{F}, \mathbf{G})$ and 1-way ANOVA with Dunnett's post hoc test (D and $\mathbf{E})$.

and CHOP protein expression by RGS2, which conflicts with our current finding (45). This discrepancy might be due to the different level of RGS2 expression depending on the inducing systems (adenoviral vector in the previous study vs. the conventional expression vector in our study) and the cell types used (normal fibroblasts in the literature vs. NSCLC cells in our study). Supporting this notion, the regulation of the UPR pathway in cancer cells was quite different from that of normal cells $(59,60)$. Further studies are required to address this important question.
The insights from our study carry important translational implications. Because various ER stresses induce the apoptosis of SCCs upon increased mRNA translation, agents that stimulate protein synthesis may facilitate the eradication of SCCs when combined with ER stress-inducing anticancer drugs, including chemotherapy. Indeed, we found that pharmacological PDE5 inhibition, which has been reported to increase protein synthesis $(46,47)$, showed the capacity to restore protein translation by activating the Akt/ mTOR/4E-BP1 pathway and sensitizing SCCs to chemotherapy, 
A

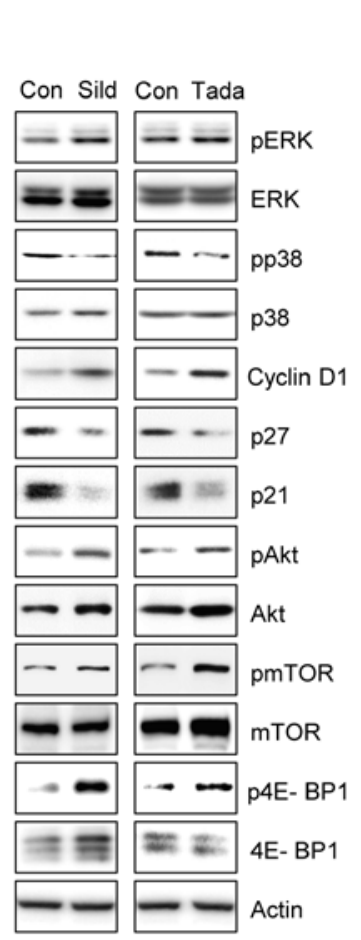

B

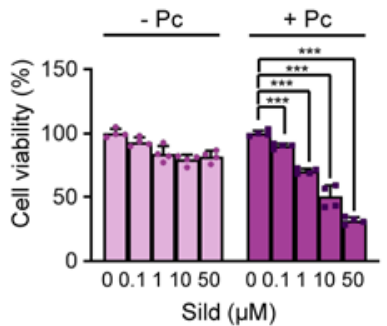

C
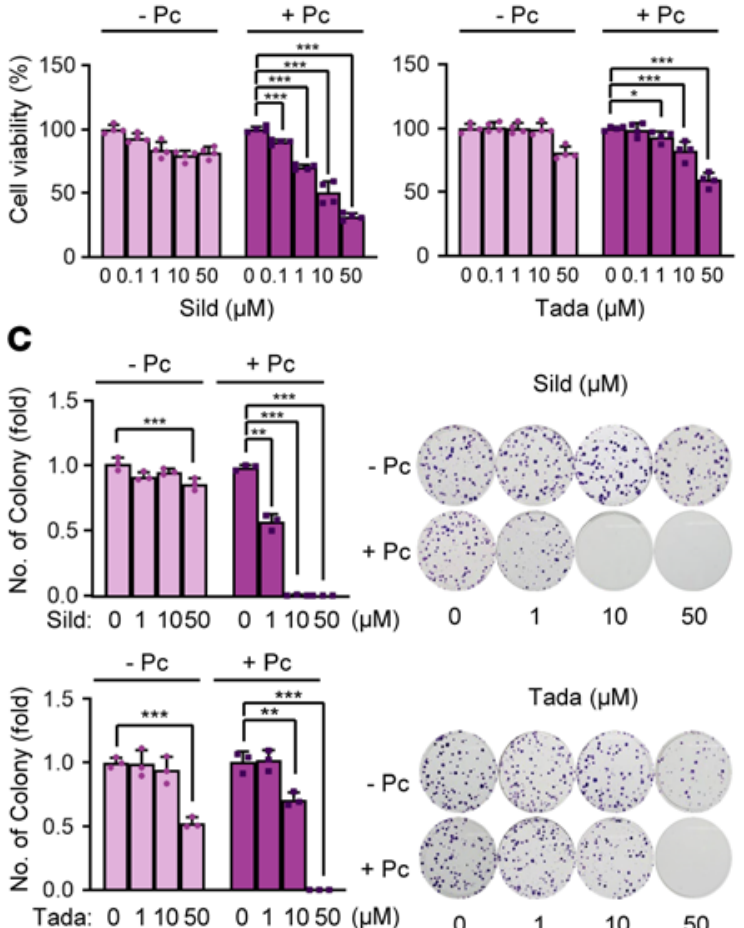

Tada $(\mu \mathrm{M})$

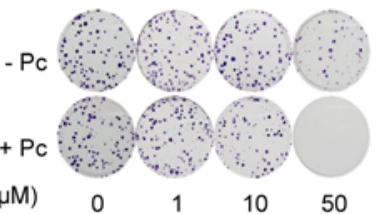

G
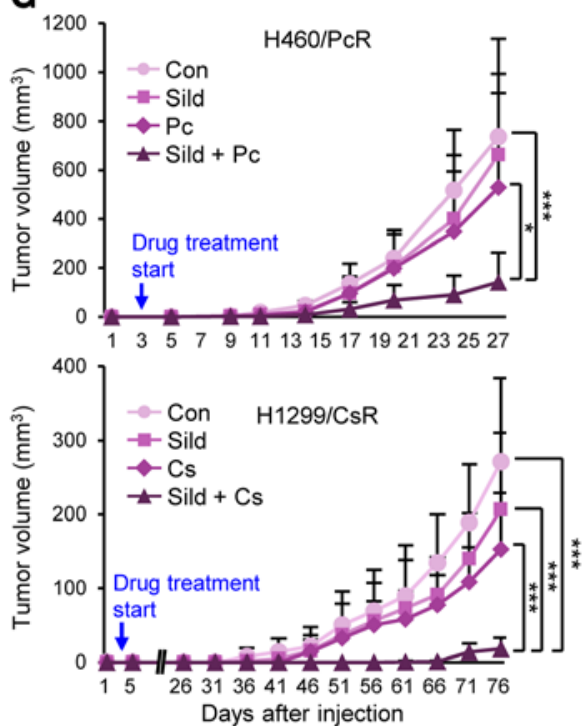

H

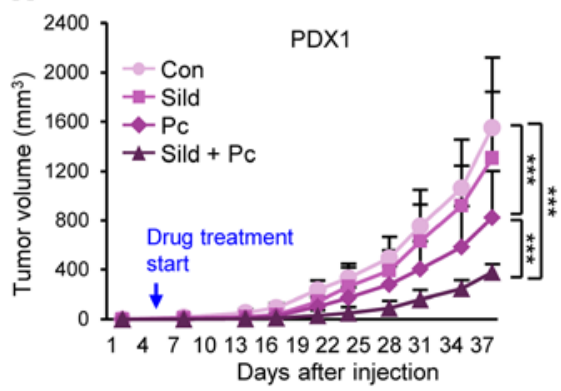

313641465156610
Days after injection

Days after injection
D
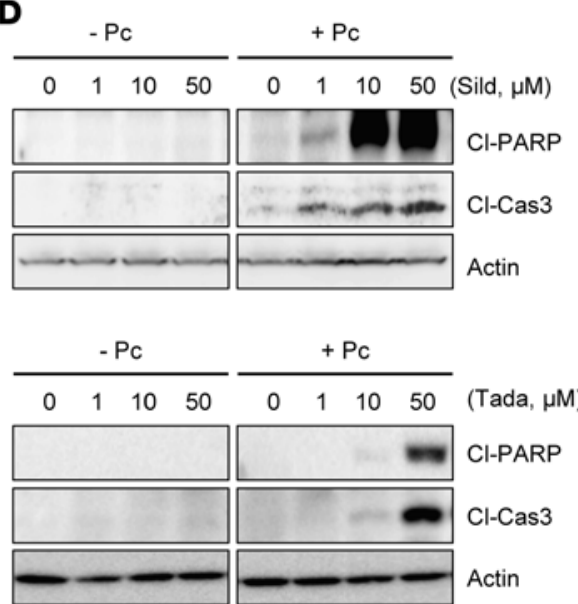

E

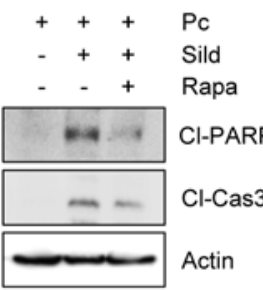

$\mathbf{F}$

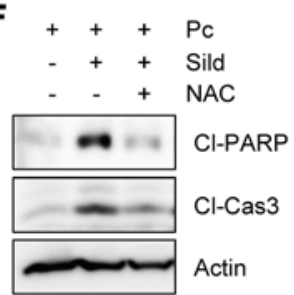

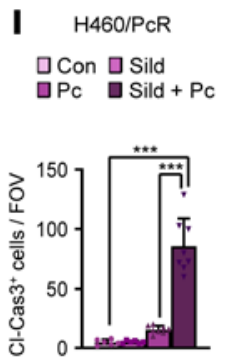

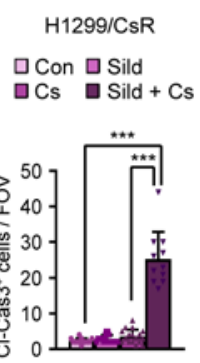

PDX1

$\square$ Con $\square$ Sild

QPc $\square$ Sild + Pc

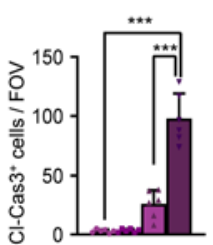

Figure 9. Chemotherapeutic sensitization via the deregulation of translational control in SCCs. (A) Immunoblotting of markers related to cell proliferation, growth, and survival in PDE5 inhibitor-treated H460/PcR cells. (B-D) Cell viability (B), anchorage-dependent colony formation (C), and apoptosis (D) in $\mathrm{H460/PcR}$ cells treated with either PDE5 inhibitor alone or in combination with paclitaxel (Pc; $20 \mathrm{nM})$. (E and F) Abrogation of sildenafil-mediated enhancement of Pc-induced apoptosis in H460/PcR cells by treatment with rapamycin (Rapa; $50 \mathrm{nM}$ ) (E) or NAC (10 mM) (F) in combination. (G-I) Potentiation of tumor growth inhibition by combined treatment with chemotherapy and sildenafil (Sild) in NOD/SCID mice bearing SCC (H460/PcR and H1299/CsR) xenografts (G) and PDX (H) (H460/PcR, Con (control): $n=6$, Sild: $n=6$, Pc: $n=8$, Sild+Pc: $n=8$; H1299/CsR, Con: $n=5$, Sild: $n=5$, cisplatin [Cs]: $n=5$, Sild+Cs: $n=6$; PDX1, $n=6$ per group). (I) IHC analyses for Cl-Cas3 in tumors treated with a combination of chemotherapy and sildenafil. The data are presented as the mean \pm SD. $n=4$ for $\mathbf{B} ; n=3$ for $\mathbf{C} ; n=6-18$ for $\mathbf{I}$. ${ }^{*} P<0.05$, ${ }^{* *} P<0.01$, and ${ }^{* * *} P<0.001$, as determined by 1 -way ANOVA with Dunnett's post hoc test.

ultimately suppressing tumor relapse. PDE5 inhibitors have been used clinically to treat erectile dysfunction and chronic obstructive pulmonary disease and exhibit an acceptable adverse effects profile $(61,62)$. More recently, the antitumor effects of PDE5 inhibitors have been investigated in preclinical and clinical settings in various types of cancers, including lung cancer (63). Previous studies have also shown the synergistic effects of PDE inhibitors with chemotherapy in lung cancer without the underlying mechanisms (64). Our current results show that repurposing PDE5 inhibitors might produce a potentially novel class of anticancer drugs by functionally targeting SCCs and thus preventing tumor relapse after chemotherapy.

In conclusion, we defined the mechanism by which local NSCLC SCCs maintain a stable, quiescence-like phenotype and survival capacity, thus contributing to cancer progression. Our data might help to develop a potentially novel SCC-targeting strategy to prevent tumor relapse in patients with NSCLC. Considering 

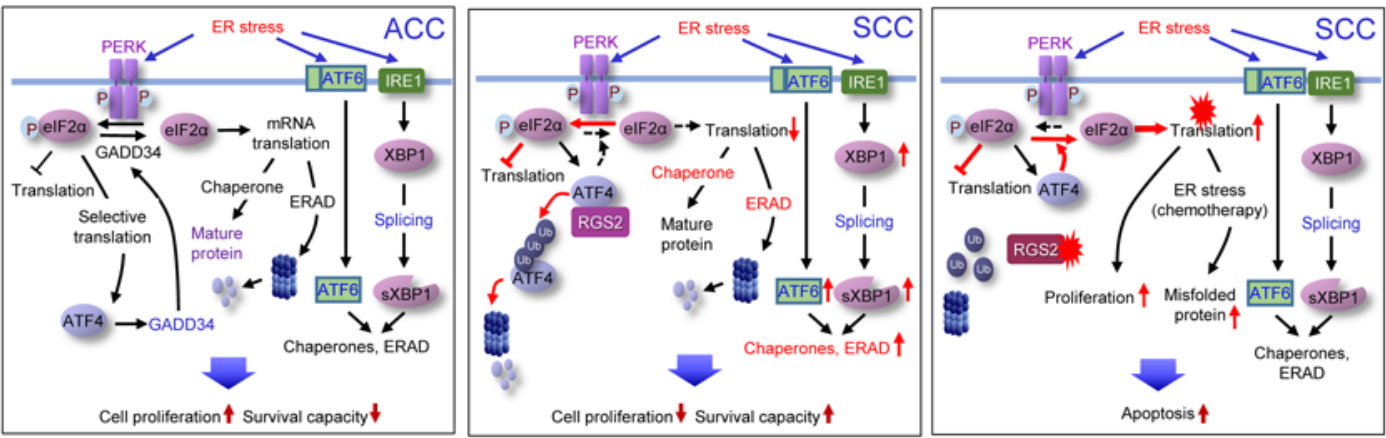

Figure 10. The proposed model describing therapeutic strategies for eradicating SCCs after chemotherapy by targeting RGS2-mediated translational control.

the heterogeneity and interpatient variability of tumors (65), further studies are warranted to address this important point.

\section{Methods}

Cell culture. Human NSCLC cell lines (H460, H1299, SK-MES-1, H226B, and H1792) and a murine lung cancer cell line (LLC) were purchased from the American Type Culture Collection or provided by John V. Heymach (MD Anderson Cancer Center, Houston, Texas, USA). Cells were cultured in RPMI 1640 medium (for human NSCLC cell lines and primary tumor cells) or DMEM (for LLC cells) supplemented with 10\% FBS and antibiotics (all from Welgene) and were maintained in a humidified atmosphere of $37^{\circ} \mathrm{C}$ with $5 \% \mathrm{CO}_{2}$. Chemotherapy-resistant cells were generated by continuous exposure to the corresponding chemotherapeutic drugs for more than 4 months. NSCLC cells and their chemotherapy-resistant subpopulations were authenticated and validated using an AmpFLSTR Identifier PCR Amplification Kit (Applied Biosystems, 4322288) in 2013, 2016, and 2017. Cells passaged for fewer than 6 months after receipt or resuscitated validated cells were used in this study.

Reagents. Antibodies against phospho-histone H3 (S10), cleaved caspase-3 (Cl-cas3), p21, LC3B, phospho-ERK (T202/Y204), ERK, phospho-eIF2 $\alpha$ (S51), PERK, ATF4, phospho-p70s6 kinase (T389), phospho-Akt (S473), Akt, phospho-4E-BP1 (S65), 4E-BP1, phospho-mTOR (S2448), and mTOR were purchased from Cell Signaling Technology. Antibodies against RGS2, Cyclin D1, CDK4, p27, Actin, eIF2 $\alpha$, GADD34, CHOP/GADD153, ubiquitin, phospho-p38 (Y182), p38, OctA-probe, and His-probe were purchased from Santa Cruz Biotechnology. Antibodies against Ki67 and PCNA were purchased from Abcam. Rabbit polyclonal anti-phospho-PERK (S713) antibody and CFSE cell division tracker kit were purchased from BioLegend. Rabbit polyclonal anti-RGS2 and anti-ATF4 antibodies were purchased from Proteintech. A mouse monoclonal anti-cleaved PARP antibody was purchased from BD Biosciences. HRP-conjugated secondary antibodies were purchased from GeneTex. Additional information on antibodies used, including source and catalog/clone number, is listed in Supplemental Table 3. Pc, sildenafil citrate, and tadalafil were purchased from Selleck Chemicals. Pm was purchased from LC Laboratories. Rapamycin was purchased from Tocris Bioscience. Cycloheximide was purchased from Merck Millipore. 3-(4,5-dimethylthiazol-2-yl)-2,5-diphenyltetrazolium bromide (MTT) was purchased from MP Biomedicals. db-cGMP was purchased from Cayman Chemical. Other chemicals, including Cs, etoposide, NAC, MG132, TG, and crystal violet, and the PKH26 Red Fluorescent Cell Linker Kit, were purchased from MilliporeSigma.
Primary culture. For primary culture of lung cancer cells from PDX tumors, tumors at a volume of about $500 \mathrm{~mm}^{3}$ were freshly isolated from NOD/SCID mice and cut with surgical scissors into small pieces. Single-cell suspensions from PDX tumors were generated using the human Tumor Dissociation Kit (Miltenyi Biotec) according to the manufacturer's instructions. Isolated cells were immediately used or cultured in RPMI 1640 medium supplemented with 10\% FBS and antibiotics for further experiments.

CFSE assay. CFSE staining was performed according to the manufacturer's instructions (BioLegend). Briefly, NSCLC cell lines or primary cells from lung PDXs $\left(1 \times 10^{6}\right.$ to $2 \times 10^{6}$ cells, each $)$ were labeled with $25 \mu \mathrm{M} \mathrm{CFSE}$ at $37^{\circ} \mathrm{C}$ for 20 minutes. Stained cells were quenched with culture media, seeded in culture plates, and then incubated for 7 days. After incubation, cultured cells were dissociated with trypsin-EDTA, and more than $3 \times 10^{7}$ single cell suspensions were prepared and sorted into CFSE $^{\text {lo }}$ (lower, $10 \%$ ), CFSE $^{\text {mid }}$ (middle, $\sim 35 \%-40 \%$ ), and CFSE ${ }^{\text {hi }}$ (upper, $10 \%$ ) populations by flow cytometry using a FACS Aria III cell sorter (BD Biosciences). The sorted cells were plated or injected immediately for subsequent in vitro or in vivo experiments. At least 3 cell sorting experiments were performed to repeat the relevant experiments.

Immunofluorescence. Cells were seeded onto coverslips and then fixed with $4 \%$ paraformaldehyde for 15 minutes at room temperature (RT). Sections (thickness: $4 \mu \mathrm{m}$ ) of FFPE tumor tissues or an NSCLC TMA including 40 tumor samples (US Biomax, Inc.) were deparaffinized, rehydrated, and then subjected to antigen retrieval (citrate buffer, pH 6.0). All samples (fixed cells or antigen-retrieved FFPE tissues and TMA) were then permeabilized with $0.2 \%$ Triton X-100 at RT and then incubated with blocking buffer (5\% normal serum in TBS containing $0.025 \%$ Triton X-100 [TBSTx]) for 1 hour at RT. Samples were incubated with the following primary antibodies: anti-Ki67 (1:1000), antiRGS2 (1:200), or anti-phospho-histone H3 (1:500), diluted in TBSTx containing 1\% BSA. After washing 3 times with TBSTx and incubating with the corresponding fluorochrome-conjugated (Alexa Fluor 488 or Alexa Fluor 594) secondary antibodies diluted in TBSTx containing $1 \%$ BSA (1:500), nuclei were counterstained with DAPI (50 ng/mL) and then coverslips were mounted with a mounting solution (Dako, Agilent). Fluorescence was observed under a confocal microscope.

RNA-Seq analysis. A library was constructed using a SENSE mRNA-Seq Library Prep Kit according to the manufacturer's recommendations. High-throughput sequencing was then performed with paired-end 100-base pair reads using a HiSeq 2000 system (Illumina). RNA-Seq reads were mapped using TopHat software to obtain 
the alignment file. The alignment file was used to assemble transcripts, estimate abundances, and detect the differential expression of genes/isoforms using Cufflinks. Library preparation and RNA-Seq were performed as next-generation sequencing (NGS) services by Ebiogen, Inc. Hierarchical clustering of RNA-Seq data and heatmap production were performed using MultiExperiment Viewer software (version 4.8.1, J. Craig Venter Institute). All sequencing data that support the findings of this study have been deposited in NCBI's Gene Expression Omnibus (GEO, GSE141218).

GO term enrichment analysis of RNA-Seq data was performed using the GO Enrichment Analysis tool available online (http:// geneontology.org/) (66). To validate the involvement of GO terms related to cell proliferation in the $\mathrm{CSFE}^{\text {hi }}$ populations, differentially regulated genes in the $\mathrm{CSFE}^{\mathrm{hi}}$ populations were compared with gene products associated with one of the following GO terms: cell cycle (GO:0007049), regulation of cell population proliferation (GO:0042127), and regulation of cell cycle (GO:0051726). The direct annotations for Homo sapiens to the GO terms described above were found using AmiGO 2 (http://amigo.geneontology.org) (67). Venn diagrams were drawn using the freely available web-based tool (http:// bioinformatics.psb.ugent.be/webtools/Venn/).

Animal studies. All animal procedures were performed using protocols approved by the Seoul National University IACUC. Mice were fed standard mouse chow and water ad libitum and housed in temperature- and humidity-controlled facilities with a 12-hour light/12hour dark cycle. For xenograft experiments, $0.5 \times 10^{6}$ to $1 \times 10^{6}$ cells, suspended in $100 \mu \mathrm{L}$ of PBS mixed with Matrigel matrix (1:1) per spot, were subcutaneously injected into the right flanks of 6-8-weekold female NOD/SCID mice. For PDX experiments, tumors that had been passaged less than 5 times in mice were minced into $2 \mathrm{~mm}^{3}$ pieces and subcutaneously implanted into 6-8-week-old female NOD/ SCID mice. After the tumor volume reached $50-100 \mathrm{~mm}^{3}$, the mice were randomly grouped and treated with either vehicle or test agents. Drugs were dissolved and administered to mice as follows. Pc was dissolved in a mixture of Cremophor EL and ethanol (1:1, v/v) and further diluted in PBS (final 1:1:18, v/v/v). Mice were i.p. treated with Pc (20 $\mathrm{mg} / \mathrm{kg}$ ) once a week. Cs and $\mathrm{Pm}$ were dissolved in $0.9 \%(\mathrm{w} / \mathrm{v}) \mathrm{NaCl}$ solution and i.p. administered once a week at a dose of $3 \mathrm{mg} / \mathrm{kg}$ and $50 \mathrm{mg} / \mathrm{kg}$, respectively. Sildenafil citrate was dissolved in $0.9 \%$ (w/v) $\mathrm{NaCl}$ solution at a dose of $10 \mathrm{mg} / \mathrm{kg}$ and orally treated 5 times in a week. Tumor growth was determined by measuring the short and long diameters of the tumors with a caliper in a blinded fashion, and body weight was measured twice weekly to monitor toxicity. Tumor volume was calculated using the following formula: tumor volume $\left(\mathrm{mm}^{3}\right)=$ $(\text { short diameter })^{2} \times($ long diameter $) \times 0.5$.

Metabolic labeling for determination of de novo ATF4 protein synthesis. Cells were incubated in methionine-free RPMI medium (Welgene) for 1 hour and pulse-labeled with $\left.0.2 \mathrm{mCi} \mathrm{L-}-{ }^{35} \mathrm{~S}\right]$ methionine (PerkinElmer) for the indicated time periods. Equal amounts of protein (1 $\mathrm{mg}$ ) from the total cell lysates were immunoprecipitated with an antiATF4 antibody (1:1000) and were then subjected to SDS-PAGE. After the gel was dried using a Model 583 gel dryer (Bio-Rad Laboratories), autoradiography was performed.

Immunoprecipitation and pulldown assay. Cells were lysed with lysis buffer (40 mM Tris- $\mathrm{HCl}$ [pH 7.4], $120 \mathrm{mM} \mathrm{NaCl,} \mathrm{0.5 \%} \mathrm{Triton}$ $\mathrm{X}-100,2 \mathrm{mM}$ EDTA, $1 \mathrm{mM} \mathrm{MgCl}_{2}$ ) containing various protease and phosphatase inhibitors (100 mM NaF, $5 \mathrm{mM} \mathrm{Na}_{3} \mathrm{VO}_{4}, 1 \mathrm{mM}$ PMSF, 1 $\mu \mathrm{g} / \mathrm{mL}$ aprotinin, $1 \mu \mathrm{g} / \mathrm{mL}$ leupeptin, and $1 \mu \mathrm{g} / \mathrm{mL}$ pepstatin). Equal amounts $(500-1000 \mu \mathrm{g})$ of cell lysates were immunoprecipitated with an anti-ATF4, anti-RGS2, or anti-flag antibody $(1 \mu \mathrm{g})$ and were then incubated with protein $\mathrm{A} / \mathrm{G}$ agarose beads at $4^{\circ} \mathrm{C}$ for 2 hours. The beads were washed 5 times with lysis buffer or PBS, and the immunoprecipitated proteins were eluted with $2 \times$ sample buffer by boiling for 5 minutes at $95^{\circ} \mathrm{C}$. The eluates were separated by $8 \%$ SDS-PAGE and further analyzed by immunoblotting.

For pulldown assays, total cell lysates were incubated with recombinant FL or truncated RGS2 proteins bound to Ni-NTA-agarose beads overnight at $4^{\circ} \mathrm{C}$. The beads were washed several times, and bound proteins were extracted as described above.

Intratumoral injection of siRNAs. The siRNA formulation for in vivo delivery using in vivo-jetPEI (Polyplus-Transfection SA) was prepared according to the manufacturer's instructions. Briefly, $5 \mu \mathrm{g}$ of siRNAs diluted in a sterile $5 \%$ glucose solution were complexed with in vivo-jetPEI at a ratio of $0.12 \mu \mathrm{L}$ of in vivo-jetPEI per $1 \mu \mathrm{g}$ of siRNAs. Formulated siRNAs were injected intratumorally twice a week.

IHC analysis. IHC analysis was performed using antibodies against cleaved caspase-3, RGS2, or ATF4. Sections of FFPE tissue specimens were deparaffinized, rehydrated, and then subjected to antigen retrieval using the citrate-based antigen unmasking solution (Vector Laboratories). After treatment with $0.3 \%$ hydrogen peroxide solution, slides were incubated with blocking buffer (5\% normal serum in TBSTx) for 1 hour at RT. Slides were incubated first with primary antibodies diluted in TBSTx containing $1 \%$ BSA (1:200) overnight at $4^{\circ} \mathrm{C}$, washed 3 times with TBSTx, and then incubated with a biotinylated secondary antibody (Vector Laboratories) diluted in TBSTx containing 1\% BSA (1:500) for 1 hour at RT. Solutions A and B (ABC-Elite, Vector Laboratories) were added simultaneously for 30 minutes, and signals were detected using a 3,3'-diaminobenzidine (DAB) substrate kit (Vector Laboratories). Slides were further counterstained with hematoxylin.

Statistics. The data are presented as the mean \pm SD. All in vitro and in vivo experiments were independently performed at least twice, and a representative result is shown. The values presented in the graphs are generated by multiple replicates in a representative experiment. No statistical methods were used to determine the sample size for in vitro and in vivo experiments. Statistical significance was determined by a 2-tailed Student's $t$ test or 1-way ANOVA using GraphPad Prism (version 7 and 8). An F-test for equality of variances was performed to ensure the same variance of 2 test groups. The Shapiro-Wilk test was performed to determine whether the in vitro or in vivo data followed a normal distribution. $P$ values less than 0.05 were considered significant.

Study approval. All animal experiments were reviewed and approved by Institutional Animal Care and Use Committee of Seoul National University and performed in compliance with institutional and governmental policies.

\section{Author contributions}

JC, HYM, HJL, JYS, MN, SHP, HJB, and RWP conducted or contributed to in vitro experiments. JC, SYH, SH, and YKS performed or contributed to in vivo experiments. JC, SJH, and HJL performed or contributed to IHC analysis. JC analyzed RNA-Seq data. HYM performed analysis of the GEO data sets. MCH provided scientific input. HYL conceived and designed the study and wrote the manuscript. 


\section{Acknowledgments}

This work was supported by a grant from the National Research Foundation of Korea (NRF), the Ministry of Science and ICT, Republic of Korea (NRF-2016R1A3B1908631).

Address correspondence to: Ho-Young Lee, Creative Research Initiative Center for Concurrent Control of Emphysema and Lung
Cancer, College of Pharmacy, Seoul National University, 1 Gwanak-ro, Gwanak-gu, Seoul 08826, Republic of Korea. Phone: 82.2.880.9277; Email: hylee135@snu.ac.kr. SJH and HJL's present address is: School of Pharmacy, Sungkyunkwan University, Suwon, Gyeonggi-do, Republic of Korea. SHP's present address is: Department of Pathology, College of Korean Medicine, Dongeui University, Busan, Republic of Korea.
1. Siegel RL, Miller KD, Jemal A. Cancer statistics, 2019. CA Cancer JClin. 2019;69(1):7-34.

2. Zarogoulidis K, et al. Treatment of non-small cell lung cancer (NSCLC). J Thorac Dis. 2013;5(suppl 4):S389-S396.

3. Gridelli C, et al. Second-line treatment of advanced non-small cell lung cancer. J Thorac Oncol. 2008;3(4):430-440.

4. Froudarakis ME, Briasoulis E. Advanced nonsmall cell lung cancer: on relapse rechallenge the tumor, not the patient. BMC Res Notes. 2010;3:195.

5. Valdes M, Nicholas G, Goss GD, Wheatley-Price P. Chemotherapy in recurrent advanced nonsmall-cell lung cancer after adjuvant chemotherapy. Curr Oncol. 2016;23(6):386-390.

6. Aguirre-Ghiso JA. Models, mechanisms and clinical evidence for cancer dormancy. Nat Rev Cancer. 2007;7(11):834-846.

7. Moore N, Houghton J, Lyle S. Slow-cycling therapy-resistant cancer cells. Stem Cells Dev. 2012;21(10):1822-1830.

8. Li LT, Jiang G, Chen Q, Zheng JN. Ki67 is a promising molecular target in the diagnosis of cancer (review). Mol Med Rep. 2015;11(3):1566-1572.

9. Sosa MS, Bragado P, Aguirre-Ghiso JA. Mechanisms of disseminated cancer cell dormancy: an awakening field. Nat Rev Cancer. 2014;14(9):611-622.

10. Sosa MS, Avivar-Valderas A, Bragado P, Wen HC, Aguirre-Ghiso JA. ERK1/ 2 and $\mathrm{p} 38 \alpha / \beta$ signaling in tumor cell quiescence: opportunities to control dormant residual disease. Clin Cancer Res. 2011;17(18):5850-5857.

11. Ranganathan AC, Adam AP, Zhang L, Aguirre-Ghiso JA. Tumor cell dormancy induced by p38SAPK and ER-stress signaling: an adaptive advantage for metastatic cells? Cancer Biol Ther. 2006;5(7):729-735.

12. Valcourt JR, Lemons JM, Haley EM, Kojima M, Demuren OO, Coller HA. Staying alive: metabolic adaptations to quiescence. Cell Cycle. 2012;11(9):1680-1696.

13. Vera-Ramirez L, Hunter KW. Tumor cell dormancy as an adaptive cell stress response mechanism. F1000Res. 2017;6:2134.

14. Avril T, Vauléon E, Chevet E. Endoplasmic reticulum stress signaling and chemotherapy resistance in solid cancers. Oncogenesis. 2017;6(8):e373.

15. Schönthal AH. Endoplasmic reticulum stress: its role in disease and novel prospects for therapy. Scientifica (Cairo). 2012;2012:857516.

16. Hetz C. The unfolded protein response: controlling cell fate decisions under ER stress and beyond. Nat Rev Mol Cell Biol. 2012;13(2):89-102.

17. Wang M, Kaufman RJ. The impact of the endoplasmic reticulum protein-folding environment on cancer development. Nat Rev Cancer. 2014;14(9):581-597.
18. Han J, et al. ER-stress-induced transcriptional regulation increases protein synthesis leading to cell death. Nat Cell Biol. 2013;15(5):481-490.

19. Ranganathan AC, Zhang L, Adam AP, Aguirre-Ghiso JA. Functional coupling of p38-induced up-regulation of $\mathrm{BiP}$ and activation of RNAdependent protein kinase-like endoplasmic reticulum kinase to drug resistance of dormant carcinoma cells. Cancer Res. 2006;66(3):1702-1711.

20. Zmijewski JW, Song L, Harkins L, Cobbs CS, Jope RS. Oxidative stress and heat shock stimulate RGS2 expression in 1321N1 astrocytoma cells. Arch Biochem Biophys. 2001;392(2):192-196.

21. Ota A, Sawai M, Sakurai H. Stress-induced transcription of regulator of $G$ protein signaling 2 (RGS2) by heat shock transcription factor HSF1. Biochimie. 2013;95(7):1432-1436.

22. Nguyen $\mathrm{CH}$, Zhao P, Sobiesiak AJ, Chidiac P. RGS2 is a component of the cellular stress response. Biochem Biophys Res Commun. 2012;426(1):129-134.

23. Kehrl JH, Sinnarajah S. RGS2: a multifunctional regulator of $\mathrm{G}$-protein signaling. Int J Biochem Cell Biol. 2002;34(5):432-438.

24. Nguyen $\mathrm{CH}$, et al. Translational control by RGS2. J Cell Biol. 2009;186(5):755-765.

25. Agarwal R, Kaye SB. Ovarian cancer: strategies for overcoming resistance to chemotherapy. Nat Rev Cancer. 2003;3(7):502-516.

26. Roesch A, et al. A temporarily distinct subpopulation of slow-cycling melanoma cells is required for continuous tumor growth. Cell. 2010;141(4):583-594.

27. Dembinski JL, Krauss S. Characterization and functional analysis of a slow cycling stem celllike subpopulation in pancreas adenocarcinoma. Clin Exp Metastasis. 2009;26(7):611-623.

28. Ebinger S, et al. Characterization of rare, dormant, and therapy-resistant cells in acute lymphoblastic leukemia. Cancer Cell. 2016;30(6):849-862.

29. Hoang-Minh LB, et al. Infiltrative and drugresistant slow-cycling cells support metabolic heterogeneity in glioblastoma. EMBO J. 2018;37(23):e98772.

30. Parish CR. Fluorescent dyes for lymphocyte migration and proliferation studies. Immunol Cell Biol. 1999;77(6):499-508.

31. Wallace PK, Tario JD, Fisher JL, Wallace SS, Ernstoff MS, Muirhead KA. Tracking antigen-driven responses by flow cytometry: monitoring proliferation by dye dilution. Cytometry $A$. 2008;73(11):1019-1034.

32. Subramanian A, et al. Gene set enrichment analysis: a knowledge-based approach for interpreting genome-wide expression profiles. Proc Natl Acad Sci USA. 2005;102(43):15545-15550.

33. Li B, et al. Fructose-1,6-bisphosphatase opposes renal carcinoma progression. Nature. 2014;513(7517):251-255.

34. Cubillos-Ruiz JR, Bettigole SE, Glimcher LH. Tumorigenic and immunosuppressive effects of endoplasmic reticulum stress in cancer. Cell. 2017;168(4):692-706.

35. Acosta JC, Gil J. Senescence: a new weapon for cancer therapy. Trends Cell Biol. 2012;22(4):211-219.

36. Sikora E, Mosieniak G, Sliwinska MA. Morphological and functional characteristic of senescent cancer cells. Curr Drug Targets. 2016;17(4):377-387.

37. Knauf U, Tschopp C, Gram H. Negative regulation of protein translation by mitogen-activated protein kinase-interacting kinases 1 and 2. $\mathrm{Mol}$ Cell Biol. 2001;21(16):5500-5511.

38. Tabas I, Ron D. Integrating the mechanisms of apoptosis induced by endoplasmic reticulum stress. Nat Cell Biol. 2011;13(3):184-190.

39. Mylonis I, Sembongi H, Befani C, Liakos P, Siniossoglou S, Simos G. Hypoxia causes triglyceride accumulation by HIF-1-mediated stimulation of lipin 1 expression. J Cell Sci. 2012;125(Pt 14):3485-3493.

40. Oslowski CM, Urano F. Measuring ER stress and the unfolded protein response using mammalian tissue culture system. Meth Enzymol. 2011;490:71-92.

41. Mahadevan NR, Zanetti M. Tumor stress inside out: cell-extrinsic effects of the unfolded protein response in tumor cells modulate the immunological landscape of the tumor microenvironment. JImmunol. 2011;187(9):4403-4409.

42. Ahmed N, Abubaker K, Findlay J, Quinn M. Epithelial mesenchymal transition and cancer stem cell-like phenotypes facilitate chemoresistance in recurrent ovarian cancer. Curr Cancer Drug Targets. 2010;10(3):268-278.

43. Pakos-Zebrucka K, Koryga I, Mnich K, Ljujic M, Samali A, Gorman AM. The integrated stress response. EMBO Rep. 2016;17(10):1374-1395.

44. Wortel IMN, van der Meer LT, Kilberg MS, van Leeuwen FN. Surviving stress: modulation of ATF4-mediated stress responses in normal and malignant cells. Trends Endocrinol Metab. 2017;28(11):794-806.

45. Wang CJ, Chidiac P. RGS2 promotes the translation of stress-associated proteins ATF 4 and CHOP via its eIF2B-inhibitory domain. Cell Signal. 2019;59:163-170.

46. Berdeaux R, Stewart R. cAMP signaling in skeletal muscle adaptation: hypertrophy, metabolism and regeneration. Am J Physiol Endocrinol Metab. 2012;303(1):E1-17.

47. Sheffield-Moore M, et al. Sildenafil increases muscle protein synthesis and reduces muscle fatigue. Clin Transl Sci. 2013;6(6):463-468.

48. Tengholm A, Gylfe E. cAMP signalling in insulin and glucagon secretion. Diabetes Obes Metab. 
2017;19(suppl 1):42-53.

49. Hahm JH, Kim S, Paik YK. Endogenous cGMP regulates adult longevity via the insulin signaling pathway in Caenorhabditis elegans. Aging Cell. 2009;8(4):473-483.

50. Hensel JA, Flaig TW, Theodorescu D. Clinical opportunities and challenges in targeting tumour dormancy. Nat Rev Clin Oncol. 2013;10(1):41-51.

51. Linder A, Hagberg Thulin M, Damber JE, Welén K. Analysis of regulator of G-protein signalling 2 (RGS2) expression and function during prostate cancer progression. Sci Rep. 2018;8(1):17259.

52. Boelte KC, Gordy LE, Joyce S, Thompson MA, Yang L, Lin PC. Rgs 2 mediates pro-angiogenic function of myeloid derived suppressor cells in the tumor microenvironment via upregulation of MCP-1. PLoS One. 2011;6(4):e18534.

53. Phi LTH, et al. Cancer stem cells (CSCs) in drug resistance and their therapeutic implications in cancer treatment. Stem Cells Int. 2018;2018:5416923.

54. Marcinak SJ, Ron D. The unfolded protein response in lung disease. Proc Am Thorac Soc. 2010;7(6):356-362.
55. Wek RC, Jiang HY, Anthony TG. Coping with stress: eIF2 kinases and translational control. Biochem Soc Trans. 2006;34(Pt 1):7-11.

56. Lu PD, Harding HP, Ron D. Translation reinitiation at alternative open reading frames regulates gene expression in an integrated stress response. JCell Biol. 2004;167(1):27-33.

57. Bi M, et al. ER stress-regulated translation increases tolerance to extreme hypoxia and promotes tumor growth. EMBO J. 2005;24(19):3470-3481.

58. Song L, Jope RS. Cellular stress increases RGS2 mRNA and decreases RGS4 mRNA levels in SH-SY5Y cells. Neurosci Lett. 2006;402(3):205-209.

59. Li X, Zhang K, Li Z. Unfolded protein response in cancer: the physician's perspective. J Hematol Oncol. 2011;4:8.

60. Walczak A, Gradzik K, Kabzinski J, Przybylowska-Sygut K, Majsterek I. The Role of the ER-Induced UPR Pathway and the Efficacy of Its Inhibitors and Inducers in the Inhibition of Tumor Progression. Oxid Med Cell Longev. 2019;2019:5729710.

61. Langtry HD, Markham A. Sildenafil: a review of its use in erectile dysfunction. Drugs. 1999;57(6):967-989.

62. Wedzicha JA, Calverley PM, Rabe KF. Roflumilast: a review of its use in the treatment of COPD. Int JChron Obstruct Pulmon Dis. 2016;11:81-90.

63. Pantziarka P, Sukhatme V, Crispino S, Bouche G, Meheus L, Sukhatme VP. Repurposing drugs in oncology (ReDO)-selective PDE5 inhibitors as anti-cancer agents. Ecancermedicalscience. 2018;12:824.

64. Domvri K, et al. Potential synergistic effect of phosphodiesterase inhibitors with chemotherapy in lung cancer. J Cancer. 2017;8(18):3648-3656.

65. Rambow F, et al. Toward minimal residual disease-directed therapy in melanoma. Cell. 2018;174(4):843-855.e19.

66. Mi H, Muruganujan A, Casagrande JT, Thomas PD. Large-scale gene function analysis with the PANTHER classification system. Nat Protoc. 2013;8(8):1551-1566.

67. Gene Ontology Consortium. Gene Ontology Consortium: going forward. Nucleic Acids Res. 2015;43(Database issue):D1049-D1056. 